\title{
A MULTIRESOLUTION APPROACH TO REGULARIZATION OF SINGULAR OPERATORS AND FAST SUMMATION*
}

\author{
GREGORY BEYLKIN ${ }^{\dagger}$ AND ROBERT CRAMER ${ }^{\dagger}$
}

\begin{abstract}
Singular and hypersingular operators are ubiquitous in problems of physics, and their use requires a careful numerical interpretation. Although analytical methods for their regularization have long been known, the classical approach does not provide numerical procedures for constructing or applying the regularized operator. We present a multiresolution definition of regularization for integral operators with convolutional kernels which are homogeneous or associated homogeneous functions. We show that our procedure yields the same operator as the classical definition. Moreover, due to the constructive nature of our definition, we provide concise numerical procedures for the construction and application of singular and hypersingular operators. As an application, we present an algorithm for fast computation of discrete sums and briefly discuss several other examples.
\end{abstract}

Key words. regularization, integral equations, integral transforms, integral operators, wavelets, multiresolution analysis, $n$-body problems

AMS subject classifications. 65R99, 70F10

PII. S1064827500379227

1. Introduction. The analytical procedure for regularization of hypersingular integrals (integrals that do not converge even as the principle value) is well understood (see, e.g., [10]). Yet there are no systematic computational methods for representing operators defined via such integrals. Recently, there has been interest in the numerical evaluation of such operators on functions. Since formal integrals defining the action of these operators are divergent, the construction of quadratures is quite delicate (see, e.g., [13]).

Our goal in this paper is to provide a multiresolution definition and, hence, regularization of such operators. Using only the degree of homogeneity of the kernel $K(x)$ and its asymptotic behavior for $|x| \rightarrow \infty$, we construct multiresolution representations of singular and hypersingular operators. In addition to serving as a definition of regularization, this representation also provides an algorithm for applying such operators to functions.

We consider operators of the form

$$
(T f)(\mathbf{x})=\int K(\mathbf{x}-\mathbf{y}) f(\mathbf{y}) d \mathbf{y}, \quad \mathbf{x}, \mathbf{y} \in \mathbb{R}^{n},
$$

where the kernel $K(\mathbf{x})$ is a homogeneous function (a precise definition is given in section 3), which may have a nonintegrable, algebraic singularity. It turns out that, once they have been described in a multiresolution setting, singular and hypersingular operators no longer require any special treatment. Therefore, such representations may provide a more convenient tool for the description of some physical phenomena than the standard integral kernels.

\footnotetext{
*Received by the editors October 6, 2000; accepted for publication (in revised form) August 28, 2001; published electronically May 20, 2002. This work was partially supported by DARPA/AFOSR grants DOD F49620-97-1-0017 and DOD F49620-98-1-0491.

http://www.siam.org/journals/sisc/24-1/37922.html

${ }^{\dagger}$ Department of Applied Mathematics, University of Colorado at Boulder, Boulder, CO 803090526 (gregory.beylkin@colorado.edu, robert.cramer@colorado.edu). The research of the first author was partially supported by DARPA/NASA grant S43 5-28646.
} 
Classically, integrals with nonintegrable singularities are given meaning by first defining the integral on test functions that vanish in a neighborhood of the singularity, then extending this definition to test functions that do not. Such a procedure is known as regularization. Typically, one considers the regularization that is "natural" in the sense that the sum of two ordinary kernels corresponds to the sum of their regularizations, the ordinary derivative of a kernel to the derivative of its regularization, and the product of the kernel with an infinitely differentiable function to the regularization of the product [10].

An effective way to arrive at the natural regularization is by analytic continuation with respect to a complex parameter $\lambda$. In this case, the original kernel is replaced by a family of kernels which are analytic with respect to $\lambda$ in some domain, say $\Lambda$, on which the kernel is locally integrable. As an example, consider the integral

$$
\int_{0}^{\infty} x^{\lambda} \phi(x) d x
$$

where $\phi$ is a smooth test function with compact support. This integral is an analytic function of $\lambda$ for $\operatorname{Re}(\lambda)>-1$ and, if the region of analyticity can be extended (we discuss this in more detail in section 2) to a wider domain, say $\Lambda_{1}$, then we define this analytic continuation to be a regularization of the integral to the domain $\Lambda_{1}$.

Although this approach leads to a consistent definition of the operator $T$, it does not provide any guidance for numerical construction of the regularized operator, nor for numerical application of the regularized operator to functions.

The results obtained via analytic continuation may also be obtained by using the Taylor expansion in the neighborhood of the singularity. For example, let $K(x)$ have a nonintegrable singularity at $x=0$. Then, for $|x-y|<\epsilon$, the function $f$ in (1) is replaced by itself minus an appropriate number of terms of its Taylor expansion, where $f$ belongs to a class of sufficiently smooth test functions. Elsewhere the operator is defined by the original integral. The regularization, if it exists, is obtained by taking a limit as $\epsilon$ goes to zero (see, e.g., [10]). Again, this approach provides no guidance to the numerical construction or application of the resulting operators.

In this paper we use multiresolution analysis (MRA) to describe an alternative regularization procedure. For homogeneous kernels we require knowledge only of the asymptotic behavior of $K(x)$ as $|x| \rightarrow \infty$, and the degree of homogeneity of $K$, to completely define the regularized operator. Besides the definition, our approach also provides a numerical method for constructing the regularization and applying the regularized operator to functions. We show that our procedure yields the same operator as the classical regularization.

We note that there are many practical applications of hypersingular integrals. For example, in electrostatics and electromagnetics, such integrals appear wherever it is necessary to work with derivatives of the Green's functions. In [13], quadrature formulas are developed for quadruple and octuple layer potentials in two dimensions, which are represented by hypersingular integrals.

One of the applications of our approach is fast evaluation of discrete sums, for example,

$$
g(x)=\sum_{n=1}^{N} \frac{f\left(x_{n}\right)}{\left(x-x_{n}\right)^{2}} .
$$

Here the function $f$ represents charges, or masses, at the particle locations $\left\{x_{n}\right\}$, and the function $g(x)$ is well defined provided that $x \neq x_{n}$ for $n=1, \ldots, N$. Such sums 
can be computed using FMM-type algorithms [11], [6]. Alternatively, the sum can be interpreted as an integral with a hypersingular kernel, regularized using our approach, then evaluated using a fast algorithm. This application is developed in section 6 of this paper.

Another application is in fluid mechanics where we encounter the projector onto spaces of divergence-free functions. The kernel of the projector is defined by

$$
K_{i j}(x)=\delta_{i j} \delta(x)-C_{n}\left[\frac{\delta_{i j}}{|x|^{n}}-\frac{n x_{i} x_{j}}{|x|^{n+2}}\right],
$$

where $C_{n}$ equals $1 / 2 \pi$ in two dimensions and $1 / 4 \pi$ in three dimensions. Direct use of this operator has largely been avoided up to now due to lack of a numerical procedure for its application. We present an example in two dimensions using this operator. The full development of this application is in progress and will be reported elsewhere.

Our approach provides an explicit representation of operators and, as a consequence, greater flexibility in applications. For example, a hypersingular kernel may be integrated over a connected region $\Omega$ in the plane, as in

$$
\int_{\Omega} K(x-y) f(y) d y
$$

or over a parameterized curve $\gamma$, as in

$$
\int_{\gamma} K(t-s) f(s) d s .
$$

Once the multiresolution regularization of the kernel $K$ has been constructed it can be used in either setting.

The remainder of this paper is organized as follows. In section 2 we provide a brief review of the classical approach to regularization of singular integrals. We begin section 3 by defining a class of operators for which our definition of regularization is well suited (namely, those which are homogeneous of some degree). We then establish some general results concerning kernels in an MRA, and finally we proceed to a multiresolution definition of regularization. Our definition is constructive and yields a practical algorithm.

The development given in section 3 uses orthogonal bases for convenience, but this is not the only type of MRA available. Thus, in section 4 we make some comments regarding the use of other bases, especially the compactly supported B-splines. In section 5 we give examples of kernels that can be regularized by the multiresolution procedure. In section 6 we develop an application, a fast algorithm for evaluation of discrete sums, such as the example given in (2).

In Appendix B we list some background material on MRA. In Appendix C we obtain an estimate on the difference between a multiresolution kernel and the singular kernel of which it is the regularization. In Appendix D we derive an alternative representation for a multiresolution kernel, namely, as a trigonometric expansion with variable coefficients. Such representation provides an efficient method for pointwise evaluation of the multiresolution kernel. The results in Appendices $\mathrm{C}$ and $\mathrm{D}$ are needed to develop the fast summation algorithm in section 6 .

2. Classical approach to regularization. Let us briefly review the classical approach to the regularization of divergent integrals. Our exposition closely follows 
[10]. The classical approach interprets a divergent integral as a functional, or generalized function, operating on a class of test functions. The origin of the mathematical treatment of generalized functions (distributions) goes back to the theory introduced by Schwartz (see, e.g., [16]). Such a functional, appropriately constructed, provides the definition for the classical regularization. We consider a natural regularization (see [10] or section 1). We note that divergent integrals involving functions with algebraic singularities are ubiquitous in physical applications. These functions increase as some power of $1 /\left|x-x_{0}\right|$, as $x$ approaches the singular point $x_{0}$, and divergent integrals involving them serve as our main examples.

As a systematic method for regularizing such integrals, we may employ the method of analytic continuation. The main idea is to construct a family of generalized functions $f_{\lambda}$ analytic with respect to a parameter $\lambda$ over some open region $\Lambda$ in the complex plane. If the functional can be extended analytically to a wider region, say $\Lambda_{1}$, then we consider the analytic continuation of the functional as a definition of the generalized function $f_{\lambda}$ for $\lambda \in \Lambda_{1}$.

We illustrate the main points with an example. Let us define $f_{\lambda}=x_{+}^{\lambda}$, where

$$
x_{+}^{\lambda}=\left\{\begin{array}{cc}
x^{\lambda}, & x>0 \\
0, & x \leq 0 .
\end{array}\right.
$$

For $\operatorname{Re}(\lambda)>-1$, this generalized function is defined by the convergent integral

$$
\left(x_{+}^{\lambda}, \phi\right)=\int_{0}^{\infty} x^{\lambda} \phi(x) d x,
$$

where $\phi(x)$ belongs to the space of infinitely differentiable test functions with compact support. Splitting the integral in (5), we redefine the functional as

$$
\left(x_{+}^{\lambda}, \phi\right)=\int_{0}^{1} x^{\lambda}[\phi(x)-\phi(0)] d x+\frac{\phi(0)}{\lambda+1}+\int_{1}^{\infty} x^{\lambda} \phi(x) d x .
$$

Observe that the first term is analytic for $\operatorname{Re}(\lambda)>-2$, the second term for $\lambda \neq-1$, and the third term for all $\lambda$. Hence, we can view this expression as an analytic continuation of the original functional to the region $\operatorname{Re}(\lambda)>-2, \lambda \neq-1$. Note also that if the test function $\phi(x)$ vanishes in a neighborhood of zero, then what remains on the right-hand side of (6) is identical to the right-hand side of (5). A complete derivation of the expressions given below is contained in Appendix A. For more details and examples, see, e.g., [10].

Let us apply the above approach to the regularization of integral operators having the general form (1). Consider the following examples with homogeneous kernels, which have an algebraic singularity at $x=y$, where we assume $f(y)$ is sufficiently differentiable and bounded as $|y| \rightarrow \infty$ :

$$
(T f)(x)=\int_{-\infty}^{\infty} \frac{f(y)}{(x-y)^{2 m+1}} d y=\int_{0}^{\infty} \frac{f(x-y)-f(x+y)}{y^{2 m+1}} d y
$$

for $m=0,1,2, \ldots$, and

$$
(T f)(x)=\int_{-\infty}^{\infty} \frac{f(y)}{(x-y)^{2 m}} d y=\int_{0}^{\infty} \frac{f(x-y)+f(x+y)}{y^{2 m}} d y
$$

for $m=1,2,3, \ldots$. These operators can be regularized by defining

$$
(T f)(x)=\int_{0}^{\infty} y^{-2 m-1}\left[f(x-y)-f(x+y)+2 \sum_{k=0}^{m-1} \frac{f^{(2 k+1)}(x)}{(2 k+1) !} y^{2 k+1}\right] d y
$$


in the first case and

$$
(T f)(x)=\int_{0}^{\infty} y^{-2 m}\left[f(x-y)+f(x+y)-2 \sum_{k=0}^{m-1} \frac{f^{(2 k)}(x)}{(2 k) !} y^{2 k}\right] d y
$$

in the second.

Remark 1 . We note that both the degree of homogeneity and the asymptotic behavior of the kernel at infinity are preserved under the regularization procedure.

The approach outlined above is a standard method for dealing with divergent integrals involving algebraic singularities. It does not, however, address the issue of numerical computation of such integrals. Although expressions (8a) and (8b) define the action of the operator on smooth functions, numerical representation of such operators remains a difficult task. The situation is different if we turn to a representation in an MRA. In this case, defining the action of the operator on scaling or wavelet functions leads to a constructive definition of the regularization of the operator, which in turn provides practical methods for computation of the regularization and for applying the operator to functions expressed in the basis.

\section{Multiresolution approach to regularization.}

3.1. Preliminary considerations. Consider a (generalized) function $K(\mathbf{x})$ and its Fourier transform

$$
\hat{K}(\xi)=\int_{-\infty}^{\infty} e^{\mathrm{i} \mathbf{x} \cdot \xi} K(\mathbf{x}) d \mathbf{x}, \quad \mathbf{x}, \xi \in \mathbb{R}^{n},
$$

where for most applications $1 \leq n \leq 3$. We assume $\hat{K}$ is homogeneous of degree $\alpha$,

$$
\hat{K}(\lambda \xi)=\lambda^{\alpha} \hat{K}(\xi), \quad \lambda>0
$$

or, equivalently,

$$
K(\lambda \mathbf{x})=\lambda^{-\alpha-n} K(\mathbf{x}) .
$$

Let us consider the integral operator $T$ with kernel $K$, defined as

$$
(T f)(\mathbf{x})=\int_{-\infty}^{\infty} K(\mathbf{x}-\mathbf{y}) f(\mathbf{y}) d \mathbf{y} .
$$

We allow $K$ to have nonintegrable singularities, in which case (11) is given meaning by regularization, and the Fourier transform (9) is understood in the sense of generalized functions (see, e.g., [10]).

Since the operator $T$ is of convolution type, we can formally express (11) in terms of the Fourier transform as

$$
(T f)(\mathbf{x})=\frac{1}{(2 \pi)^{n}} \int_{-\infty}^{\infty} e^{-\mathbf{i} \mathbf{x} \cdot \xi} \hat{K}(\xi) \hat{f}(\xi) d \xi .
$$

In the nonintegrable case this integral is also given meaning by regularization.

To simplify notation we describe our approach in a one-dimensional setting. However, all considerations below can be extended to multiple dimensions with little difficulty by using the tensor product construction (see Appendix B). We indicate how this is done and in section 6 provide a numerical example in two dimensions. 
We begin by assuming that integrals in (11) and (12) are convergent. With this assumption we derive a system of equations that allows us to compute the projection of the operator onto an MRA without evaluating any integrals. The computation requires knowledge only of the degree of homogeneity and values of the kernel $K(x)$ for large $|x|$. If integrals in (11) and (12) are not convergent, then we use this construction as a definition of multiresolution regularization.

3.1.1. Test functions. The subspaces $\left\{V_{j}\right\}$ of an MRA (see Appendix B) serve as the spaces of test functions. Let $\phi(x)$ be the scaling function for the MRA. The functions

$$
\left\{\phi_{j, k}(x)=2^{-j / 2} \phi\left(2^{-j} x-k\right) \mid k \in \mathbb{Z}\right\}
$$

form an orthonormal basis for the subspace $V_{j}$.

We make extensive use of the two-scale difference equation (see Appendix B)

$$
\phi_{j, k}(x)=\sum h_{l} \phi_{j-1,2 k+l}(x) .
$$

In what follows, we consider projection of a kernel onto the MRA and show that (13) leads to a two-scale difference equation for the coefficients of the projection which, for homogeneous kernels, relates coefficients on the same scale. This relationship, together with asymptotic behavior of the kernel $K(x)$ for $|x| \rightarrow \infty$, provides the means for obtaining the coefficients of the projection.

Formally applying the operator $T$ to the basis function $\phi_{j, k}$ we obtain the following proposition.

Proposition 1. An operator $T$ with kernel homogeneous of degree $\alpha$ scales as

$$
\left(T \phi_{j, k}\right)(x)=2^{-\alpha j}(T \phi)_{j, k}(x) .
$$

Proof. Rearranging (12) we have

$$
(T f)(x)=\frac{1}{2 \pi} \int_{-\infty}^{\infty} \hat{K}(\xi) \int_{-\infty}^{\infty} f(y) e^{-i \xi(x-y)} d y d \xi
$$

Using this expression, we have

$$
\begin{aligned}
\left(T \phi_{j, k}\right)(x) & =\frac{1}{2 \pi} \int_{-\infty}^{\infty} \hat{K}(\xi) \int_{-\infty}^{\infty} 2^{-j / 2} \phi\left(2^{-j} y-k\right) e^{-i \xi(x-y)} d y d \xi \\
& =\frac{1}{2 \pi} \int_{-\infty}^{\infty} \hat{K}(\xi) \int_{-\infty}^{\infty} 2^{j / 2} \phi(y) e^{-i 2^{j} \xi\left(2^{-j} x-k-y\right)} d y d \xi \\
& =\frac{1}{2 \pi} \int_{-\infty}^{\infty} \hat{K}\left(2^{-j} \xi\right) \int_{-\infty}^{\infty} 2^{-j / 2} \phi(y) e^{-i \xi\left(2^{-j} x-k-y\right)} d y d \xi \\
& =2^{-\alpha j} 2^{-j / 2}(T \phi)\left(2^{-j} x-k\right),
\end{aligned}
$$

where we used (10a).

3.1.2. Multiresolution representation of the operator. Let us construct a sequence of operators $T_{j}$ such that $T_{j}: V_{j} \rightarrow V_{j}$ and the kernel of $T_{j}$ has the form

$$
T_{j}(x, y)=\sum_{m} \sum_{n} t_{m-n}^{j} \phi_{j, m}(x) \phi_{j, n}(y), \quad j \in \mathbb{Z},
$$


where $\left\{t_{n}^{j} \mid n \in \mathbb{Z}\right\}$ are coefficients on scale $j$. For an operator with a homogeneous kernel, the projections $T_{j}$ are related to each other by scaling.

Proposition 2. The projections $T_{j}$ of an operator $T$ with kernel homogeneous of degree $\alpha$ scale as

$$
T_{j}(x, y)=2^{-\alpha j} 2^{-j} T_{0}\left(2^{-j} x, 2^{-j} y\right)
$$

where

$$
T_{0}(x, y)=\sum_{m} \sum_{n} t_{m-n} \phi(x-m) \phi(y-n),
$$

and $\left\{t_{n} \mid n \in \mathbb{Z}\right\}$ are coefficients on scale $j=0$,

$$
t_{n}=\int \phi_{0, n}(x)\left(T \phi_{0,0}\right)(x) d x .
$$

Proof. To establish (16), we consider the coefficients in (15), defined as

$$
t_{m-n}^{j}=\int \phi_{j, m}(x)\left(T \phi_{j, n}\right)(x) d x .
$$

Using (14) we have

$$
\begin{aligned}
t_{m-n}^{j} & =2^{-\alpha j} \int \phi_{j, m}(x)(T \phi)_{j, n}(x) d x \\
& =2^{-\alpha j} 2^{-j} \int \phi\left(2^{-j} x-m\right)(T \phi)\left(2^{-j} x-n\right) d x \\
& =2^{-\alpha j} \int \phi(x-(m-n))(T \phi)(x) d x \\
& =2^{-\alpha j} t_{m-n}^{0} .
\end{aligned}
$$

Thus,

$$
t_{n}^{j}=2^{-\alpha j} t_{n}
$$

and by substituting this expression into (15), we obtain (16).

Remark 2. Equation (19) is independent of the number of dimensions. For example, in two dimensions, using the tensor product basis (see Appendix B), we have

$$
t_{m, n}^{j}=2^{-\alpha j} t_{m, n} .
$$

3.1.3. Two-scale difference equation for the coefficients. In order to compute the coefficients $\left\{t_{n}\right\}$ in (17) that represent an operator with a homogeneous kernel, we utilize the following condition which is necessary for the existence of these coefficients.

Proposition 3. Let the coefficients $\left\{t_{n}\right\}$ represent an operator with kernel homogeneous of degree $\alpha$. If these coefficients exist, then they must satisfy the two-scale difference equation

$$
2^{-\alpha} t_{n}=\sum_{m=-m_{0}}^{m_{0}} a_{m} t_{2 n+m},
$$


where

$$
a_{m}=\sum_{k} h_{k} h_{k+m}
$$

and $\left\{h_{k}\right\}$ are the coefficients in (13).

Proof. First note that $\phi(x)=\sum h_{l} \phi_{-1, l}(x)$ from (13), and this, together with (14), implies

$$
(T \phi)(x)=\sum_{l} h_{l}\left(T \phi_{-1, l}\right)(x)=2^{\alpha} \sum_{l} h_{l}(T \phi)_{-1, l}(x) .
$$

Using this expression we have

$$
\begin{aligned}
t_{n} & =\int \phi(x-n)(T \phi)(x) d x \\
& =\int \sum_{k} h_{k} \phi_{-1,2 n+k}(x) 2^{\alpha} \sum_{l} h_{l}(T \phi)_{-1, l}(x) d x \\
& =2^{\alpha} \sum_{k} \sum_{l} h_{k} h_{l} \int \phi(2 x-2 n-k)(T \phi)(2 x-l) 2 d x \\
& =2^{\alpha} \sum_{m}\left(\sum_{k} h_{k} h_{k+m}\right) \int \phi(x-(2 n+m))(T \phi)(x) d x \\
& =2^{\alpha} \sum_{m} a_{m} t_{2 n+m},
\end{aligned}
$$

which establishes (20).

Remark 3. Using (92)-(94) of Appendix B, we can express (20) as

$$
2^{-\alpha} t_{n}=t_{2 n}+\sum_{m=0}^{\left(m_{0}-1\right) / 2} a_{2 m+1}\left(t_{2 n-2 m-1}+t_{2 n+2 m+1}\right) .
$$

In two dimensions, we have

$$
\begin{aligned}
2^{-\alpha} t_{n, n^{\prime}}=t_{2 n, 2 n^{\prime}} & +\sum_{m, m^{\prime}} a_{2 m+1} a_{2 m^{\prime}+1}\left(t_{2 n-2 m-1,2 n^{\prime}-2 m^{\prime}-1}+t_{2 n+2 m+1,2 n^{\prime}-2 m^{\prime}-1}\right. \\
& \left.+t_{2 n-2 m-1,2 n^{\prime}+2 m^{\prime}+1}+t_{2 n+2 m+1,2 n^{\prime}+2 m^{\prime}+1}\right) .
\end{aligned}
$$

This pattern is clear and repeats in any number of dimensions.

Remark 4. Equations (21) and (22) contain infinitely many unknowns. However, we show in the following subsection that all but a small number of these are determined by asymptotic behavior of the kernel $K(x)$ for large $|x|$.

3.2. Multiresolution definition of regularization. Let us now change the point of view. We no longer assume that integrals defining the operator are convergent. Instead, we use (14)-(20) to construct the representation of an operator with a nonintegrable kernel.

Given an integral operator with a homogeneous kernel, our construction uses the two-scale difference equation (20), together with knowledge of the asymptotic behavior of the kernel for large $|x|$, to compute the coefficients $\left\{t_{n} \mid n \in \mathbb{Z}\right\}$ that represent the operator in an MRA. The result is a constructive regularization of such operators. 
Multiresolution regularization is consistent with the classical definition. As previously noted, the classical regularization alters neither the degree of homogeneity of the kernel nor its asymptotic behavior at infinity, and we use only these two properties to uniquely determine the multiresolution regularization. The relationship between classical and multiresolution regularization is discussed more fully in section 3.4.

There are three steps in our construction.

Step 1 . We assume the coefficients $\left\{t_{n}\right\}$ in (18) are known for large $|n|$. Indeed, for sufficiently large $|n|$, the integrals defining $t_{n}$ are convergent since the domain of integration does not contain the singularity of the kernel $K(x)$. As a practical matter, we assume the asymptotic condition

$$
t_{n}=F(n)+O\left(\frac{1}{n^{2 M+1+\alpha}}\right) \quad \text { as }|n| \rightarrow \infty
$$

holds with some function $F$. The parameter $M$ in the exponent on the left-hand side is typically the number of vanishing moments associated with the chosen MRA (see Appendix B). We show in section 3.3 how to find $F(n)$.

Using (23), we determine all coefficients $t_{n}$ for large $|n|$. Given $\epsilon>0$, we choose a positive integer $n_{0}$ sufficiently large to ensure that $\left|t_{n}-F(n)\right|<\epsilon$ if $|n| \geq n_{0}$; then we define

$$
t_{n}=F(n) \quad \text { for }|n| \geq n_{0} .
$$

Step 2. We use the two-scale difference equation

$$
2^{-\alpha} t_{n}=\sum_{-m_{0}}^{m_{0}} a_{m} t_{2 n+m}
$$

to compute $\left\{t_{n}\right\}$ for $m_{0}<|n|<n_{0}$. Since $|n|>m_{0}$, the right-hand side of (24) does not involve the coefficient $t_{n}$ and thus provides an explicit expression for $t_{n}$ in terms of $t_{2 n-m_{0}}, \ldots, t_{2 n+m_{0}}$.

Step 3 . We solve the linear system defined by (24) for coefficients in the range $|n| \leq m_{0}$. Coefficients in this range appear on both sides of the two-scale difference equation. Let us express this linear system with matrix notation as

$$
2^{-\alpha} \mathbf{t}=A \mathbf{t}+\mathbf{b}
$$

where $\mathbf{t}$ represents the vector $\left\{t_{-m_{0}}, \ldots, t_{m_{0}}\right\}$. The matrix elements are $A_{i j}=a_{2 i-j}$, where $\left\{a_{m}\right\}$ are the coefficients in (24) and, thus, are determined by the choice of the basis. Entries of the vector $\mathbf{b}$ consist of products of the coefficients $\left\{a_{m}\right\}$ with the known coefficients $t_{n}$ for $|n|>m_{0}$ and therefore contain information obtained from the asymptotic condition $F(n)$ in (23).

We are now in a position to give a constructive definition of the multiresolution regularization of the operator $T$, but let us first consider eigenvalues and eigenvectors of the matrix $A$ in (25).

3.2.1. Generalized kernels concentrated on a discrete set of points. The following proposition is well known.

Proposition 4 (see, e.g., [10]). Any generalized kernel $K(x)$ concentrated on a discrete set of points is a finite linear combination of the delta function and its derivatives. 
It follows that a generalized kernel homogeneous of (fixed) degree $k=0,1,2, \ldots$ has the general form (see, e.g., [10])

$$
K(x)=\frac{c_{1}}{x_{+}^{k+1}}+\frac{c_{2}}{x_{-}^{k+1}}+C \delta^{(k)}(x),
$$

where $c_{1}, c_{2}$, and $C$ are constants and $x_{+}$and $x_{-}$are defined in Appendix A.

If the kernel $K(x)$ is $\delta^{(k)}(x)$, then the operator $T$ is simply $k$ th derivative. This case has been considered in [4], where it was shown that if the wavelet basis has a sufficient number of vanishing moments, then the two-scale difference equation (25) reduces to

$$
2^{-k} \mathbf{t}=A \mathbf{t}
$$

plus an additional normalization condition. Thus, in this case, $\mathbf{t}$ is the eigenvector of the matrix $A$.

We also have the following proposition.

Proposition 5 (see, e.g., [9]). Let the matrix $A$ be defined as in (25), and let $M$ be the number of vanishing moments in the MRA. Then, for $k=0,1, \ldots, 2 M-1$, $2^{-k}$ is an eigenvalue of $A$.

It has been shown (see [14]) that eigenvalue 1 , corresponding to $k=0$, must be simple. Eigenvalues $2^{-k}$ for $k=1,2, \ldots$ may have higher multiplicities depending on the choice of the basis.

In what follows, we assume that the kernel has no components which are concentrated on a discrete set of points, and, hence, the solution $\mathbf{t}$ of (25) has no projection onto eigenvectors of $A$.

3.2.2. Definition of multiresolution regularization. We now present our definition.

DeFinition 6. Let $T$ be an operator with the kernel $K(x)$, homogeneous of degree $\alpha$, and with no component concentrated on a discrete set of points. Provided the solution $\mathbf{t}$ to the system of linear equations (25) exists, we obtain the multiresolution kernel $T_{0}(x, y)$, with coefficients $\left\{t_{n}\right\}$, via Steps 1-3 above. We define the operators $T_{j}: V_{j} \rightarrow V_{j}, j \in \mathbb{Z}$, with kernels $T_{j}(x, y)=2^{-\alpha j} 2^{-j} T_{0}\left(2^{-j} x, 2^{-j} y\right)$, to be the multiresolution regularization of the operator $T$ on the chosen MRA.

There are three possibilities:

1. If $2^{-\alpha}$ is not an eigenvalue of $A$, then the unique solution is simply

$$
\mathbf{t}=\left(2^{-\alpha} I-A\right)^{-1} \mathbf{b} .
$$

2. If $2^{-\alpha}$ is an eigenvalue of $A$, then let $\left(2^{-\alpha} I-A\right)^{\dagger}$ denote the generalized inverse, and let $P$ denote the orthogonal projection onto the range of $\left(2^{-\alpha} I-A\right)$. If $P \mathbf{b}=\mathbf{b}$, then the solution is

$$
\mathbf{t}=\left(2^{-\alpha} I-A\right)^{\dagger} \mathbf{b}
$$

This solution is unique since, due to our assumptions, $\mathbf{t}$ has no projection onto the eigenvectors of $A$.

3. If $P \mathbf{b} \neq \mathbf{b}$, then a consistent multiresolution regularization does not exist.

Remark 5. If the regularization exists, then it is clear that two operators are the same if their kernels have the same degree of homogeneity, and the same asymptotic behavior for large $|x|$. Thus, representations of singular and hypersingular operators with homogeneous kernels are fully defined by these two attributes. 
Example 1. The simplest example of an MRA is the Haar system. The autocorrelation coefficients for this system are $a_{0}=1, a_{1}=a_{-1}=1 / 2$, and $a_{m}=0$ if $|m|>1$. The number of vanishing moments is $M=1$. For this MRA, the linear system of equations $(25)$ is

$$
2^{-\alpha}\left[\begin{array}{c}
t_{-1} \\
t_{0} \\
t_{1}
\end{array}\right]=\left[\begin{array}{ccc}
1 / 2 & 0 & 0 \\
1 / 2 & 1 & 1 / 2 \\
0 & 0 & 1 / 2
\end{array}\right]\left[\begin{array}{c}
t_{-1} \\
t_{0} \\
t_{1}
\end{array}\right]+\left[\begin{array}{c}
(1 / 2) t_{-3}+t_{-2} \\
0 \\
t_{2}+(1 / 2) t_{3}
\end{array}\right],
$$

where $t_{-3}, t_{-2}, t_{2}, t_{3}$ can be determined with any desired accuracy $\epsilon$ from the asymptotic condition (23). For example, we have

$$
\begin{aligned}
& 2^{-\alpha} t_{2}=t_{4}+\frac{1}{2}\left(t_{3}+t_{5}\right), \\
& 2^{-\alpha} t_{3}=t_{6}+\frac{1}{2}\left(t_{5}+t_{7}\right),
\end{aligned}
$$

and so on. Eigenvalues for this matrix are $\{1,1 / 2,1 / 2\}$.

Example 2. If $\alpha=0$, then the eigenvalue is 1 , and the solution is

$$
\left[\begin{array}{c}
t_{-1} \\
t_{0} \\
t_{1}
\end{array}\right]=\left[\begin{array}{ccc}
4 / 3 & -2 / 3 & -2 / 3 \\
0 & 0 & 0 \\
-2 / 3 & -2 / 3 & 4 / 3
\end{array}\right]\left[\begin{array}{c}
(1 / 2) t_{-3}+t_{-2} \\
0 \\
t_{2}+(1 / 2) t_{3}
\end{array}\right]
$$

provided that

$$
\left[\begin{array}{ccc}
2 / 3 & -1 / 3 & -1 / 3 \\
-1 / 3 & 2 / 3 & -1 / 3 \\
-1 / 3 & -1 / 3 & 2 / 3
\end{array}\right]\left[\begin{array}{c}
(1 / 2) t_{-3}+t_{-2} \\
0 \\
t_{2}+(1 / 2) t_{3}
\end{array}\right]=\left[\begin{array}{c}
(1 / 2) t_{-3}+t_{-2} \\
0 \\
t_{2}+(1 / 2) t_{3}
\end{array}\right] .
$$

If $K(x)=x^{-1}$, then we have

$$
t_{n}=(n+1) \ln \left(1+\frac{1}{n}\right)+(n-1) \ln \left(1-\frac{1}{n}\right)
$$

for $|n| \geq 2$, which clearly satisfies (27). The explicit solution for $|n| \leq 1$ is

$$
\left[\begin{array}{lll}
t_{-1} & t_{0} & t_{1}
\end{array}\right]^{T}=\left[\begin{array}{lll}
-2 \ln 2 & 0 & 2 \ln 2
\end{array}\right]^{T}
$$

which agrees with the value of the integral defining $t_{n}$ (see section 3.3), which is convergent in this case.

The following result can be of help in determining whether or not a multiresolution regularization exists for a given operator.

Proposition 7. Let $M$ be the number of vanishing moments in the MRA. For $\alpha=0,1,2, \ldots, 2 M-1$, a necessary condition for existence of a solution $\mathbf{t}$ to the linear system (25) is

$$
\sum_{i=1-2 M}^{2 M-1} i^{\alpha} b_{i}=0
$$

where $b_{i}$ is the ith element of $\mathbf{b}$.

Proof. It can be shown (see, e.g., [9]) that there exists a left eigenvector of $A$, say $\mathbf{x}$, belonging to eigenvalue $2^{-\alpha}$ for $\alpha=0,1,2, \ldots, 2 M-1$, which has the form

$$
\mathbf{x}=\left\{i^{\alpha}\right\}_{i=1-2 M}^{2 M-1} .
$$


If $\mathbf{t}$ satisfies (25), then we have

$$
\begin{aligned}
& 2^{-\alpha} \mathbf{t}=A \mathbf{t}+\mathbf{b} \\
& \Longrightarrow \quad 2^{-\alpha} \mathbf{x}^{T} \mathbf{t}=\left(\mathbf{x}^{T} A\right) \mathbf{t}+\mathbf{x}^{T} \mathbf{b} \\
& \Longrightarrow \quad 0=\mathbf{x}^{T} \mathbf{b} \text {, }
\end{aligned}
$$

which implies (28).

3.3. Asymptotic condition for integral operators. Let us establish the asymptotic condition (23). The coefficients $\left\{t_{n}\right\}$ are defined formally by

$$
t_{n}=\int \phi(x-n)(T \phi)(x) d x,
$$

and thus

$$
t_{n}=\int \phi(x-n) \int K(x-y) \phi(y) d y d x .
$$

Changing the order of integration, we have

$$
t_{n}=\int K(x) \Phi(x-n) d x,
$$

where $\Phi(x)$ is the autocorrelation of the scaling function $\phi(x)$ (see Appendix B).

Since $\Phi(x)$ is compactly supported it follows that, for sufficiently large $|n|$, the integral in (29) converges. Moreover, since $K(x)$ is smooth over the domain of integration (see (26)), the integral may be evaluated to arbitrary accuracy using a suitable quadrature. With an orthonormal system the quadrature rule is especially simple since, in this case, the autocorrelation has vanishing moments (see Appendix B), namely,

$$
\int x^{m} \Phi(x) d x=\delta_{m, 0} \quad \text { for } m=0,1, \ldots, 2 M-1,
$$

where $M$ is the number of vanishing moments in the MRA. It follows that, for large $|n|$, the one-point quadrature $t_{n} \approx K(n)$ provides satisfactory results.

Proposition 8. If the kernel is homogeneous of degree $\alpha$, then

$$
t_{n}=K(n)+O\left(\frac{1}{n^{2 M+1+\alpha}}\right)
$$

for $|n|$ sufficiently large.

Proof. Assume that the domain of integration in (29) does not contain the singularity of $K$. Then we can substitute the Taylor expansion of $K$ about $n$ into the integral to obtain

$$
\begin{aligned}
t_{n} & =\sum_{m=0}^{2 M-1} \frac{K^{(m)}(n)}{m !} \int(x-n)^{m} \Phi(x-n) d x+\int \frac{K^{(2 M)}\left(x_{0}\right)}{(2 M) !}(x-n)^{2 M} \Phi(x-n) d x \\
& =K(n)+\int \frac{K^{(2 M)}\left(x_{0}\right)}{(2 M) !}(x-n)^{2 M} \Phi(x-n) d x,
\end{aligned}
$$


where we have used (30). Thus,

$$
t_{n}-K(n)=\int \frac{K^{(2 M)}\left(x_{0}\right)}{(2 M) !}(x-n)^{2 M} \Phi(x-n) d x
$$

where $x_{0}$ lies between $x$ and $n$. The assertion (31) now follows upon demonstrating that the integral on the right, divided by $1 / n^{2 M+1+\alpha}$, is bounded for all sufficiently large $|n|$.

Differentiating (10b) repeatedly, we obtain

$$
x^{2 M} K^{(2 M)}(x)=(\alpha+1)(\alpha+2) \cdots(\alpha+2 M) K(x),
$$

and combining this with the explicit form of $K(x)$ given in (26), we obtain

$$
\frac{K^{(2 M)}\left(x_{0}\right)}{(2 M) !}=\frac{C}{x_{0}^{2 M+1+\alpha}}
$$

where $C$ is a constant. Thus,

$$
n^{2 M+1+\alpha} \int \frac{K^{(2 M)}\left(x_{0}\right)}{(2 M) !}(x-n)^{2 M} \Phi(x-n) d x=C \int \frac{(x-n)^{2 M}}{\left(x_{0} n^{-1}\right)^{2 M+1+\alpha}} \Phi(x-n) d x,
$$

which is clearly bounded for $|n|$ sufficiently large.

3.4. Relationship between multiresolution and classical regularization. In section 3.3 we showed that the coefficients $\left\{t_{n}\right\}$ may be defined formally by

$$
t_{n}=\int K(x) \Phi(x-n) d x .
$$

However, it is necessary to use a regularization procedure to define $t_{n}$ if this integral is divergent. We have achieved this using the multiresolution approach, but if the autocorrelation $\Phi(x)$ has enough derivatives, then this could also be accomplished using the classical approach outlined in section 2. Let us demonstrate that the classical approach to regularization of (32) yields the same operator as the multiresolution approach.

By way of example, let us assume that $K(x)=|x|^{-\alpha-1}$, where $2 m-2<\alpha<2 m$ for some positive integer $m$. We then use (76b) of Appendix A to redefine (32) as

$$
t_{n}=\int_{0}^{\infty} x^{-\alpha-1}\left[\Phi(n-x)+\Phi(n+x)-2 \sum_{k=0}^{m-1} \frac{\Phi^{(2 k)}(n)}{(2 k) !} x^{2 k}\right] d x
$$

This integral converges for all $n$ if $\Phi(x)$ has continuous derivatives up through order $2 m$. (For $K(x)=\operatorname{sgn}(x)|x|^{-\alpha-1}$, with $2 m-1<\alpha<2 m+1$, we use (76a) of Appendix A, provided $\Phi(x)$ has continuous derivatives up through order $2 m+1$.) To show that the coefficients $\left\{t_{n}\right\}$ defined by (33) are identical to those produced by the multiresolution regularization procedure, it is sufficient to show that, for large $|n|$, coefficients $\left\{t_{n}\right\}$ in (33) and (23) are the same, and that the two-scale difference equation (24) is satisfied.

Due to compact support, $\Phi(n+x)$ and $\Phi^{(2 k)}(n)$ vanish for all sufficiently large $|n|$, and the above expression reduces to

$$
t_{n}=\int_{0}^{\infty} \frac{\Phi(x-n)}{x^{\alpha+1}} d x
$$


where we have taken into account that $\Phi$ is an even function. Hence, as Proposition 8 shows, coefficients in (33) agree within prescribed accuracy with the coefficients used to initialize the regularization procedure in (23).

To see that coefficients $\left\{t_{n}\right\}$ in (33) satisfy the two-scale difference equation (24), note that $\Phi$ satisfies

$$
\Phi(x)=\sum_{m=-m_{0}}^{m_{0}} a_{m} \Phi(2 x+m)
$$

(see Appendix B) and, assuming that $\Phi$ is sufficiently differentiable, we also have

$$
\Phi^{(2 k)}(x)=2^{2 k} \sum_{m=-m_{0}}^{m_{0}} a_{m} \Phi^{(2 k)}(2 x+m) \quad \text { for } k=1, \ldots, m-1 .
$$

Coefficients $\left\{a_{m}\right\}$ in the above equations are identical to those in (24). Substituting these two-scale difference equations into (33), we have

$$
\begin{aligned}
t_{n} & =\int_{0}^{\infty} x^{-\alpha-1} \sum_{j} a_{j}\left[\Phi(2 n+j-2 x)+\Phi(2 n+j+2 x)-2 \sum_{k=0}^{m-1} \frac{\Phi^{(2 k)}(2 n+j)}{(2 k) !}(2 x)^{2 k}\right] d x \\
& =2^{\alpha} \sum_{j} a_{j} \int_{0}^{\infty} x^{-\alpha-1}\left[\Phi(2 n+j-x)+\Phi(2 n+j+x)-2 \sum_{k=0}^{m-1} \frac{\Phi^{(2 k)}(2 n+j)}{(2 k) !} x^{2 k}\right] d x,
\end{aligned}
$$

which is equivalent to

$$
2^{-\alpha} t_{n}=\sum_{j} a_{j} t_{2 n+j}
$$

Thus, we have the following proposition.

Proposition 9. Let the kernel $K(x)$ be homogeneous of degree $\alpha$ and with no component concentrated on a discrete set of points. Assume an orthonormal basis of compactly supported scaling functions, and such that the autocorrelation $\Phi$ has a sufficient number of continuous derivatives. Then the coefficients $\left\{t_{n}\right\}$ obtained via multiresolution regularization represent the same operator as that defined by the classical regularization.

Remark 6 . The above argument demonstrates that our construction is consistent with the classical regularization, which further implies that our construction is independent of the choice of basis (although for different bases we would of course obtain different coefficients).

3.5. Nonstandard form. We note that if the operators $T_{j}$ on all scales $j \in \mathbb{Z}$ are available, then the nonstandard form is also available (see [5]). The nonstandard form consists of triplets $\left\{A_{j}, B_{j}, \Gamma_{j}\right\}$, on all scales $j \in \mathbb{Z}$, where

$$
A_{j}: W_{j} \rightarrow W_{j}, \quad B_{j}: V_{j} \rightarrow W_{j}, \quad \Gamma_{j}: W_{j} \rightarrow V_{j},
$$

and where $W_{j}=V_{j-1}-V_{j}$ are the wavelet spaces associated with the chosen MRA. In terms of the projection operators $P_{j}: L^{2}(\Re) \rightarrow V_{j}$ and $Q_{j}=P_{j-1}-P_{j}$, we have

$$
A_{j}=Q_{j} T_{j-1} Q_{j}, \quad B_{j}=Q_{j} T_{j-1} P_{j}, \quad \Gamma_{j}=Q_{j} T_{j-1} P_{j}, \quad T_{j}=P_{j} T_{j-1} P_{j} .
$$


For example, to compute the coefficients $\left\{\alpha_{n}\right\}$ of $A_{0}$, we have the formal integral

$$
\alpha_{n}=\int \psi(x-n)(T \psi)(x) d x=\int \psi(x-n) \int K(x-y) \psi(y) d y d x,
$$

where $\psi(x)$ is the wavelet associated with the chosen MRA. Using the two-scale equation

$$
\psi(x)=\sqrt{2} \sum_{l} g_{l} \phi(2 x-l)
$$

we obtain

$$
\alpha_{n}=2^{\alpha} \sum_{m} t_{2 n+m}\left(\sum_{k} g_{k} g_{k+m}\right),
$$

where $\left\{t_{n}\right\}$ are the coefficients of the kernel $T_{0}$, and $\alpha$ is the degree of homogeneity of $K$. As in (19), we have

$$
\alpha_{n}^{j}=2^{-\alpha j} \alpha_{n}
$$

for coefficients $\left\{\alpha_{n}^{j}\right\}$ of $A_{j}$. Similar expressions hold for coefficients of $B_{j}$ and $\Gamma_{j}$.

In [5] it is shown that the nonstandard form fully describes a bounded CalderonZygmund operator and, in particular, it implies that the sequence $T_{j}$ converges to $T$ as $j \rightarrow-\infty$.

4. Remarks on using B-splines or other bases. In the previous section we considered multiresolution regularization using compactly supported, orthonormal bases involving a single scaling function. However, for practical application, we may prefer to use other bases - for example, bases of multiwavelets (see [1] and [2]). Such bases involve several compactly supported scaling functions, and we will consider applications using them elsewhere.

We may also choose to use a biorthogonal system, which involves a pair of dual scaling functions (see [8]). If both scaling functions are compactly supported, then there is no difference from the case of a single scaling function described above. If one of the scaling functions is not compactly supported, then some changes have to be introduced into the construction, and we indicate below how to handle this case using the B-splines as an example.

The B-splines do not form an orthogonal basis but may be considered as part of a biorthogonal system (see, e.g., [7], [8]). Let us choose a dual scaling function that forms a basis for the same subspace as that spanned by the B-splines but is not compactly supported. This leads to an infinite matrix in the regularization procedure (see (25)). To avoid working with such matrices, we can reduce the problem to that with a finite matrix by using the compactly supported B-splines to regularize the operator and, afterwards, change the basis (as necessary) to obtain the desired result.

Let $\beta(x)$ denote the central B-spline of odd degree (we use splines of odd degree for convenience). If the degree is $M-1$, where $M$ is an even integer, then the support of $\beta(x)$ is $\{x:|x| \leq M / 2\}$. For easy evaluation, we find it advantageous to represent kernels exclusively in terms of the compactly supported B-splines and, thus, would like to end up with regularized kernels of the form

$$
T_{0}(x, y)=\sum_{k} \sum_{l} t_{k-l} \beta(x-k) \beta(y-l) .
$$


Let $\gamma(x)$ denote the dual scaling function, constructed to satisfy

$$
\int \beta(x) \gamma(x-n) d x=\delta_{n, 0}, \quad n \in \mathbb{Z} .
$$

It follows that the coefficients $\left\{t_{n}\right\}$ in (34) are defined formally by

$$
t_{n}=\int \gamma(x-n)(T \gamma)(x) d x
$$

Since the dual scaling function is not compactly supported, $\gamma(x-n)$ fails to vanish in a neighborhood of the singularity of the kernel of $T$, and the integral in (35) may fail to converge for all integers $n$. On the other hand, to start the regularization procedure, it is necessary to assign values to the coefficients $t_{n}$ for large $|n|$ in a consistent manner. In addition, the two-scale difference equation satisfied by $\gamma$ contains infinitely many fully coupled nonzero coefficients.

Rather than working with $\gamma$ directly, let us begin instead by considering the following expression, which is dual to (34):

$$
T_{0}(x, y)=\sum_{j} \sum_{k} \tau_{j-k} \gamma(x-j) \gamma(y-k),
$$

where the coefficients $\left\{\tau_{n}\right\}$ are defined formally by

$$
\tau_{n}=\int \beta(x-n)(T \beta)(x) d x .
$$

If the coefficients $\left\{\tau_{n}\right\}$ are available, then, since the dual scaling function $\gamma$ can be expressed in terms of $\beta$, the coefficients $\left\{t_{n}\right\}$ in (34) can be obtained directly from them.

Due to compact support of $\beta(x)$, integrals in (37) are convergent for all sufficiently large $|n|$, and we are able to compute coefficients $\tau_{n}$ directly from this integral expression to provide the necessary starting point for the regularization procedure. Equation (37) can be expressed as

$$
\tau_{n}=\int_{-M}^{M} B(x) K(x+n) d x,
$$

where $B(x)=\int \beta(x+y) \beta(y) d y$ is the autocorrelation of $\beta(x)$, which is supported on the interval $\{x:|x| \leq M\}$. For $|n|>M$, this integral is convergent and can be evaluated using any suitable quadrature. For example, we can define

$$
\tau_{n}=M \sum_{j=1}^{N} w_{j} B\left(M x_{j}\right) K\left(M x_{j}+n\right), \quad|n|>M,
$$

where $\left\{x_{j}\right\}$ are Gaussian nodes and $\left\{w_{j}\right\}$ are Gaussian weights. (Alternatively, since $B(x)$ is a piecewise polynomial and $K(x)$ is of the form $1 / x^{1+\alpha}$ (see (26)), explicit formulas can be worked out for the integrals in (38).) In addition, coefficients $\left\{\tau_{n}\right\}$ satisfy a two-scale difference equation with a finite number of coefficients, namely,

$$
2^{-\alpha} \tau_{n}=\frac{1}{2^{2 M-1}} \sum_{k=0}^{2 M}\left(\begin{array}{c}
2 M \\
k
\end{array}\right) \tau_{2 n+k-M}, \quad n \in \mathbb{Z} .
$$


Thus, the regularization procedure described in section 3 can be used, with (39) and (40) in place of (23) and (24), respectively, to obtain all coefficients $\left\{\tau_{n}\right\}$ that define the kernel (36).

Once we have the coefficients $\left\{\tau_{n}\right\}$ in (36), the regularized kernel $T_{0}(x, y)$ is known. Since $\gamma$ can be expressed as a linear combination of B-splines, we have

$$
\gamma(x)=\sum_{-\infty}^{\infty} q_{j} \beta(x-j),
$$

which can be used to perform the change of basis from (36) to (34). Substituting (41) into (35), we obtain

$$
t_{n}=\sum_{m} \tau_{m} \tilde{q}_{n-m}
$$

where

$$
\tilde{q}_{m}=\sum_{k} q_{k} q_{m+k}
$$

In the Fourier domain, functions $\beta$ and $\gamma$ are related via

$$
\hat{\gamma}(\xi)=\frac{\hat{\beta}(\xi)}{a(\xi)}
$$

where $a(\xi)$ is a trigonometric polynomial (see, e.g., [7]). Taking Fourier transforms on both sides of (41), we observe that the coefficients $\left\{q_{j}\right\}$ are Fourier coefficients of the function $1 / a(\xi)$, and the coefficients $\left\{\tilde{q}_{m}\right\}$ in (42) are Fourier coefficients of the function $1 /[a(\xi)]^{2}$. If we define trigonometric series $\hat{t}$ and $\hat{\tau}$ by

$$
\hat{t}(\xi)=\sum_{-\infty}^{\infty} t_{n} e^{i n \xi} \quad \text { and } \quad \hat{\tau}(\xi)=\sum_{-\infty}^{\infty} \tau_{n} e^{i n \xi},
$$

then we can express (42) as

$$
\hat{t}(\xi)=\frac{\hat{\tau}(\xi)}{[a(\xi)]^{2}} .
$$

5. Examples. In addition to kernels which are homogeneous of some degree, we can also use our methodology to regularize the so-called associated homogeneous kernels. In this section, we give a definition of these kernels and indicate how the regularization procedure can be modified to include them. We also mention some further examples of homogeneous kernels.

5.1. Homogeneous functions. If $K(x)$ is a one-dimensional, homogeneous kernel, then, as defined in (10a), the Fourier transform satisfies

$$
\hat{K}(\lambda \xi)=\lambda^{\alpha} \hat{K}(\xi), \quad \lambda>0,
$$

which implies that

$$
\hat{K}(\xi)=c_{1} \xi_{+}^{\alpha}+c_{2} \xi_{-}^{\alpha}
$$


(see Appendix A for definition of $\xi_{+}$and $\xi_{-}$). Note that the derivative of the delta function also satisfies (43) but, as explained in section 3.2.1, we exclude this case from consideration. If $\alpha=0,1,2, \ldots$, then the case of $c_{1}=(i)^{\alpha+1} \pi / \alpha !=-c_{2}$ in (44) corresponds to the convolutional kernels with algebraic singularities

$$
K(x)=1 / x^{1+\alpha}, \quad \alpha=0,1,2, \ldots
$$

For example, the kernel of the Hilbert transform in the Fourier domain is $\hat{K}(\xi)=$ $-i \operatorname{sgn}(\xi)$, which we obtain from (44) by taking $\alpha=0$, with $c_{+}=-i$ and $c_{-}=i$.

The general form of a homogeneous function in $n$ dimensions (without a deltafunction component) may be given as

$$
\hat{K}(\vec{\xi})=F\left(\omega_{1}, \ldots, \omega_{n}\right) r^{\alpha},
$$

where $r^{2}=\xi_{1}^{2}+\cdots+\xi_{n}^{2}$, and $\omega_{i}=\xi_{i} / r$. Thus, $F$ is defined on the unit sphere in $\mathbb{R}^{n}$.

5.2. Associated homogeneous functions. From (43), it follows that the function $\hat{K}$ must satisfy the following differential equation:

$$
\xi \frac{d \hat{K}}{d \xi}=\alpha \hat{K}
$$

Thus $\hat{K}$ is an eigenfunction of the operator $(\xi \cdot d / d \xi)$ belonging to eigenvalue $\alpha$. If $\hat{K}_{0}$ satisfies (46), then we may define a sequence $\hat{K}_{1}, \hat{K}_{2}, \ldots$ of associated homogeneous functions as follows:

$$
\begin{aligned}
& \left(\xi \frac{d}{d \xi}-\alpha\right) \hat{K}_{0}=0 \\
& \left(\xi \frac{d}{d \xi}-\alpha\right) \hat{K}_{1}=\hat{K}_{0} \\
& \left(\xi \frac{d}{d \xi}-\alpha\right) \hat{K}_{2}=\hat{K}_{1}
\end{aligned}
$$

It can be shown (see, e.g., [10]) that $\hat{K}_{1}$ must satisfy

$$
\hat{K}_{1}(\lambda \xi)=\lambda^{\alpha} \hat{K}_{1}(\xi)+\left(\lambda^{\alpha} \log \lambda\right) \hat{K}_{0}(\xi)
$$

and the solution to this equation is

$$
\hat{K}_{1}(\xi)=\log |\xi| \hat{K}_{0}(\xi)+c_{1} \xi_{+}^{\alpha}+c_{2} \xi_{-}^{\alpha} .
$$

For example, if $\hat{K}_{0}=1$ and $c_{1}=c_{2}=0$ in (47), then we obtain

$$
\hat{K}_{1}(\xi)=\log \xi .
$$

To regularize such kernels using the multiresolution procedure, it is necessary only to modify the two-scale difference equation (24), since the coefficients $\left\{t_{n}\right\}$ representing $\hat{K}_{1}(\xi)$ in an MRA satisfy the two-scale difference equation

$$
2^{-\alpha} t_{n}=\sum_{m} a_{m} t_{2 n+m}+\left(2^{-\alpha} \log 2\right) \tilde{t}_{n}
$$

where $\left\{\tilde{t}_{n}\right\}$ are the coefficients representing $\hat{K}_{0}(\xi)$.

Associated homogeneous functions in multiple dimensions can be defined analogously (see, e.g., [10]). 

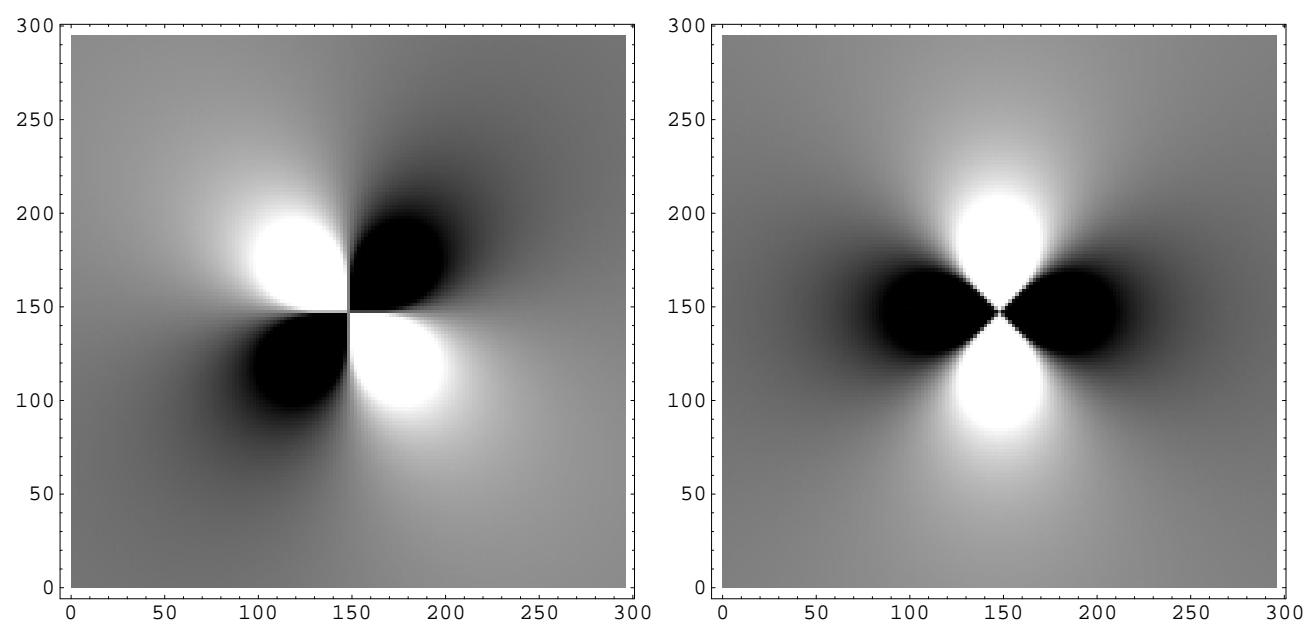

FIG. 5.1. The projector onto divergence-free functions in two dimensions. We display only two of the four kernels $K_{i j}$, since the other two are related by symmetry. The wavelet representation of these kernels will have significant coefficients only near the singularity.

5.3. Further examples. An example in $n=2$ or $n=3$ dimensions is the projector onto spaces of divergence-free functions, which is defined by

$$
K_{i j}(x)=\delta_{i j} \delta(x)-C_{n}\left[\frac{\delta_{i j}}{|x|^{n}}-\frac{n x_{i} x_{j}}{|x|^{n+2}}\right],
$$

where $C_{2}=1 / 2 \pi$ and $C_{3}=1 / 4 \pi$. In the Fourier domain, this operator corresponds to multiplication by

$$
\hat{K}_{i j}(\vec{\xi})=\delta_{i j}-\frac{\xi_{i} \xi_{j}}{|\vec{\xi}|^{2}}
$$

where $\xi_{i} /|\xi|$ is the kernel of the Riesz transform. We note that the first and second terms are both homogeneous of degree zero, but with different asymptotics. The coefficient matrix representing this operator has been computed using the approach of this paper and is displayed in Figure 5.1. Since there are many applications of this operator, we will address its construction and use in detail elsewhere.

The class of homogeneous and associated homogeneous kernels also includes freespace Green's functions for the Poisson problem in two and three dimensions, namely, $K(x, y)=\log \left(x^{2}+y^{2}\right)^{-1 / 2}$ and $K(x, y, z)=\left(x^{2}+y^{2}+z^{2}\right)^{-1 / 2}$.

The expression (see, e.g., [12])

$$
\Phi(r, \phi, \theta)=\frac{1}{4 \pi} \int \Phi\left(r^{\prime}, \phi^{\prime}, \theta^{\prime}\right) \frac{r^{\prime}\left(r^{2}-r^{\prime 2}\right)}{\left(r^{2}+r^{\prime 2}-2 r r^{\prime} \cos \gamma\right)^{3 / 2}} d \Omega^{\prime}
$$

which involves a homogeneous kernel, gives the electrostatic or gravitational potential outside a sphere of radius $r^{\prime}$ in terms of the potential on the surface of the sphere, where $\gamma$ is the angle between vectors whose coordinates are $(r, \phi, \theta)$ and $\left(r^{\prime}, \phi^{\prime}, \theta^{\prime}\right)$. 
6. Fast summation of discrete sums. In this section we develop an application, namely, a method for fast summation of discrete sums of the form

$$
g_{i}=\sum_{\substack{j=1 \\ j \neq i}}^{N} K\left(\mathbf{x}_{i}-\mathbf{x}_{j}\right) f_{j},
$$

where $\mathbf{x}_{i} \in \mathbb{R}^{n}$ are particle locations, and $f_{i}$ is the charge carried by the $i$ th particle. The kernel $K(\mathbf{x})$ is a homogeneous function which describes interparticle interactions. For vector-valued kernels we apply the algorithm in each index.

Particle models are frequently encountered in the computer study of physical systems. Among numerous examples are $N$-body simulations in astrophysics and vortex methods in fluid mechanics. In many such models evaluation of a discrete sum, which accounts for pairwise interaction between particles, is the most expensive part of the computation. To account for the pairwise interactions directly requires $O\left(N^{2}\right)$ operations for an $N$-particle system. In this section, we present an algorithm to accomplish this in $O(N+N \log N)$ operations.

The basic computational problem in particle models may be viewed as that of computing the value, at each particle location, of the potential field generated by the particle ensemble, while excluding the self-interaction which is generally infinite. To provide background, we mention two algorithms, namely, the fast multipole method (FMM) and the method of local corrections (MLC). The FMM (see, e.g., [11]) has been highly successful in constructing fast algorithms for a variety of summation problems and incorporates several ideas which are common to such algorithms. The MLC [3] was introduced as a vortex method for problems in fluid mechanics, though the main ideas are applicable in a wider context.

After discussing these two algorithms, we present an algorithm based on multiresolution regularization (section 3), which we compare to FMM and MLC. Our approach may be viewed as similar to either of these, depending on how we choose to apply the multiresolution kernel. Choosing Fourier transform methods produces an algorithm similar to MLC, but we can also exploit the wavelet decomposition and the nonstandard form [5], which produces an algorithm similar to FMM.

Remark 7. We note that "modern" FMM (see, e.g., [6]) uses approximations with exponentials, which significantly improves its efficiency. The incorporation of similar approximations into our algorithm is in progress and will be reported elsewhere.

For simplicity, we describe the earliest version of FMM. In this approach, a sum of the form (48) is expanded as a Laurent series, or multipole expansion. At points distant from the particle ensemble, the expansion takes the form of a rapidly converging power series, and this far-field potential is well approximated by only a few terms of the expansion. If the number of terms required to achieve the desired accuracy is less than the number of particles, then evaluating the multipole expansion requires less effort than evaluating the sum (48), and a significant increase in computational speed may be realized. This method of computing the far-field potential is the basic mechanism for gaining computational efficiency in the FMM.

To exploit this mechanism, a hierarchical subdivision of space into boxes on several scales is constructed, which induces a subdivision of the particle ensemble into subcollections. By introducing several scales into the model, computation of the interaction between different subcollections can be performed on a scale at which they are well separated, which allows for use of the far-field expansions.

Beginning with the finest scale, a multipole expansion is constructed for each box at each level of the hierarchy, which represents the far-field potential due to the 
particles in the box. Expansions on coarser levels are obtained by merging expansions at the next finer level. After completing this step, the far-field interactions can be computed for each box.

Beginning with the coarsest scale, interactions between well-separated boxes are computed using the multipole expansions. These contributions are accumulated in the form of a multipole expansion for each box, which is then translated to the box subdivisions on the next finer scale. This procedure is repeated until a multipole expansion has been constructed for each box in the hierarchy, which represents the far-field potential due to the particle subcollections in all well-separated (exterior) boxes.

The final step in the FMM is to compute all near-field particle interactions directly. Since the near-field potential at each particle location involves only a few neighboring particles, the number of operations required for this step is a constant times $N$, where $N$ is the number of particles and the constant is small relative to $N$.

The MLC also seeks to evaluate a sum of the form (48), which represents the velocity field induced by an ensemble of point vortices. The velocity field of a point vortex becomes unbounded near the vortex center but is smooth elsewhere. Thus, in MLC as in FMM, the basic strategy is to approximate the velocity field of a point vortex at distant points by polynomials, while using an explicit formula for points of the field near the vortex center.

MLC begins by constructing an approximation to the velocity field at each point of an equally spaced grid overlaying the computational domain. This construction involves solution of a discretized Laplace equation and is extended from the grid to the vortex centers by a polynomial interpolating function. The number of operations needed for constructing and evaluating the approximate velocity field is proportional to $M \log M$, where $M$ is the number of grid points.

Each point vortex is approximated by a radially symmetric function with finite support, called a "vortex blob," and the approximation to the velocity field of a vortex blob agrees closely with the actual velocity at points sufficiently far from the vortex center, but diverges from the correct velocity near the center. This implies that the approximation to the total velocity field, evaluated at a vortex center, contains the correct contribution from distant vortices, but the contribution from nearby vortices is in error. Since the distance between vortex centers is measured relative to the grid spacing, one can always rescale to make all vortex centers well separated, but this is generally inefficient. A more efficient strategy is to correct the initial approximation at each vortex center to remove errors due to nearby vortices.

To correct the initial approximations, the MLC first computes the contribution to the approximate velocity field due to nearby vortices, then subtracts this quantity and adds the correct contribution obtained from an explicit formula. As in the FMM, this last step involves only a few nearby vortices for each vortex center, and thus the number of operations required for this step is a constant times $N$, where $N$ is the total number of vortex blobs and the constant is small relative to $N$.

Remark 8. We note that MLC does not obtain an explicit representation of the correction operator, which is done in our approach (see section 6.4). Such explicit representations are sometimes useful, especially if the problem is not restricted to evaluating sums.

6.1. Multiresolution algorithm for fast summation. We use the methods for regularizing singular and hypersingular operators described above to develop an algorithm for fast computation of the vector $\left\{g_{1}, \ldots, g_{N}\right\}$, where $g_{i}$ is defined by (48). 
Due to the nature of the kernel $K(x)$ in (48), the potential field of a particle is easily approximated by smooth functions at points sufficiently distant from the particle locations, a situation similar to that encountered in our discussion of FMM and MLC above. The main difference in our approach is that instead of using polynomials to approximate the far-field of a point charge (or point vortex), we use the multiresolution regularization of the kernel $K$ on the scale $j$ (see section 3.2), denoted by $T_{j}(x, y)$.

As in the MLC, the multiresolution algorithm consists of two steps: an approximation step and a correction step. In the approximation step, we replace the kernel $K(x-y)$ in (48) by its multiresolution regularization $T_{j}(x, y)$ and perform the summation. We choose to carry out the summation using an FFT, and thus the number of operations required for this step is proportional to $M \log M$, where $M$ is a gridsize determined by the scale of the projection. Alternatively, this operation could be carried out using the nonstandard form of the kernel $T_{j}$ (see [5]).

It is shown in Appendix $\mathrm{C}$ that the kernel $K(x-y)$ is well approximated by $T_{j}(x, y)$ if and only if the distance $|x-y|$ is sufficiently large. Thus, in replacing $K$ by $T_{j}$ in (48), we have introduced significant errors only for interactions between pairs of particles that are not well separated, while interactions between well-separated particles have been computed to within the desired precision (analogous to the situation with MLC).

We can always choose a scale of resolution so fine that all pairs of particles in the ensemble are well separated, since a multiresolution regularization is easily rescaled (see (16)). However, choosing ever finer scales is generally not an efficient strategy, because the number of grid points eventually becomes large enough to degrade performance. As in the MLC we perform a second step to correct the errors in the initial approximation due to particles that are close together. For each particle, we compute the contribution to the initial approximation due to nearby particles, then subtract this quantity and add the correct contribution obtained using the original kernel $K$. In contrast to the MLC, we obtain an explicit representation of the correction operator. For each particle, this step involves only a few nearby particles, and thus the computational cost of this step is a constant times $N$, where $N$ is the total number of particles and the constant is small relative to $N$.

As already mentioned, we could also apply the multiresolution regularization via the nonstandard form [5], although we do not demonstrate this in the present work. In this case we would not need a "correction step," and the algorithm would more closely resemble the FMM.

Remark 9. We begin by describing the fast summation algorithm in a onedimensional setting, and then discuss the higher-dimensional implementation. Onedimensional formulas are readily transformed into higher-dimensional formulas, using the tensor product construction (see Appendix B), by simply treating all real scalar variables as vectors and integer indices as multi-indices.

Although the derivation does not change in two dimensions there is one important additional feature used: for a wide class of problems, the correction operator has a low separation rank (up to chosen precision). This allows us to use singular value decomposition of the coefficient matrix to significantly increase the speed of evaluating the correction operator.

6.2. "Reverse discretization" of the sum. To make use of the regularization technique developed in section 3, we interpret (48) as an integral operator by interpreting the numbers $\left\{g_{m}\right\}$ as values of a function $g(x)$ defined by

$$
g(x)=\int K(x-y) f(y) d y
$$


Thus, $g_{m}=g\left(x_{m}\right)$, and, defining $f$ by

$$
f(x)=\sum_{n=1}^{N} f_{n} \delta\left(x-x_{n}\right),
$$

we recover the discrete sum (48). Moreover, (49) has the form of (11), and we can apply the regularization algorithm of section 3.2 to compute the multiresolution regularizations $T_{j}, j \in \mathbb{Z}$, of this operator.

6.3. Approximation step. In this step, we replace the kernel $K(x-y)$ in (48) by its multiresolution regularization $T_{j}(x, y)$ and perform the summation. The summation is carried out using an FFT, so that the computational effort required for this step is proportional to $M \log M$, where $M$ is a gridsize determined by the scale of the projection. We note that replacing the singular kernel with its multiresolution regularization in (48) introduces a finite self-interaction, which is removed at the correction step (section 6.4).

Let us substitute $T_{j}(x, y)$ for $K(x-y)$ on the right-hand side of (48) and denote the resulting left-hand side as $g_{j, m}$. We obtain

$$
g_{j, m}=\sum_{n=1}^{N} T_{j}\left(x_{m}, x_{n}\right) f_{n}=\sum_{k} \hat{s}_{k}^{j} \phi_{j, k}\left(x_{m}\right),
$$

where

$$
\hat{s}_{k}^{j}=\sum_{l} t_{k-l}^{j} s_{l}^{j}
$$

and

$$
s_{l}^{j}=\sum_{n=1}^{N} f_{n} \phi_{j, l}\left(x_{n}\right) .
$$

The quantities $\left\{s_{l}^{j}\right\}$ are coefficients of the projection of the particle ensemble onto the basis and are equivalent to

$$
s_{l}^{j}=\int f(x) \phi_{j, l}(x) d x,
$$

with $f$ defined by (50). The quantities $\left\{\hat{s}_{k}^{j}\right\}$ are coefficients of the projection of the potential field generated by the particle ensemble. The operation indicated in (52) is an application of a Toeplitz matrix to a vector, which is accomplished using an FFT. The matrix entries are $\left\{t_{k-l}^{j}\right\}$, which are the coefficients that represent the regularized kernel.

6.4. Correction step. In this step, we correct errors contained in the initial approximations (51). These errors exist because the regularization $T_{j}(x, y)$ cannot provide a good approximation to the original kernel $K(x-y)$ near the singularity at $x=y$, that is, for particles that are close together.

It is shown in Appendix $\mathrm{C}$ that, for any $\epsilon>0$, there exists a constant $B_{j}(\epsilon)$ such that

$$
\left|K(x-y)-T_{j}(x, y)\right|<\epsilon \quad \text { if }|x-y|>B_{j} .
$$


It is therefore necessary to correct only those contributions due to particles that are closer together than $B_{j}$. To accomplish this, we subtract the erroneous contribution from the nearby particles and add the correct contribution. Thus, for each $m=$ $1, \ldots, N$, for all $n$ such that $0<\left|x_{m}-x_{n}\right| \leq B_{j}$, we perform the following operation,

$$
g_{j, m} \longleftarrow g_{j, m}+\left[K\left(x_{m}-x_{n}\right)-T_{j}\left(x_{m}, x_{n}\right)\right] f_{n} .
$$

The self-interaction is also removed during this stage.

The values $T_{j}\left(x_{m}, x_{n}\right)$ of the multiresolution regularization are computed according to the following (already truncated) expansion:

$$
T_{j}\left(x_{m}, x_{n}\right)=2^{-j(\alpha+1)}\left\{I_{0}+2 \sum_{k=1}^{3} I_{k} \cos \left(2^{-j} k \pi\left(x_{m}+x_{n}\right)\right)\right\},
$$

where

$$
I_{k}=I_{k}\left(2^{-j}\left(x_{m}-x_{n}\right)\right)
$$

for each $k$. (See Appendix D for a complete derivation of (56).) The expansion decays rapidly, and truncation after four terms preserves accuracy roughly equal to the number of vanishing moments in the chosen MRA. Functions $I_{0}, I_{1}, I_{2}$, and $I_{3}$ are tabulated and stored in memory, and cubic polynomial interpolation is sufficient to approximate these functions with double precision. Thus, in total, roughly twenty operations are required to evaluate $T_{j}\left(x_{m}, x_{n}\right)$ in (56).

Our final expression for the approximations $\left\{g_{j, m}\right\}$ is

$$
\begin{aligned}
g_{j, m}= & \left(\sum_{n=1}^{N} T_{j}\left(x_{m}, x_{n}\right) f_{n}\right)-T_{j}\left(x_{m}, x_{m}\right) f_{m} \\
& +\sum_{\substack{\left|x_{m}-x_{n}\right|<B_{j} \\
m \neq n}}\left[K\left(x_{m}-x_{n}\right)-T_{j}\left(x_{m}, x_{n}\right)\right] f_{n} .
\end{aligned}
$$

Using the estimate derived in Appendix C, it is easily verified that

$$
\max _{1 \leq m \leq M}\left|g_{j, m}-g_{m}\right|<\epsilon \sum\left|f_{n}\right| .
$$

6.5. An example in one dimension. Let the kernel $K$ be defined by

$$
K(x-y)=1 /(x-y), \quad x \neq y .
$$

The discrete sums to be computed are

$$
g_{m}=\sum_{\substack{n=1 \\ n \neq m}}^{N} \frac{f_{n}}{x_{m}-x_{n}}
$$

for $m=1, \ldots, N$. The kernel is homogeneous of degree zero and has the Fourier transform $\hat{K}(\xi)=i \pi \operatorname{sgn} \xi$.

In Tables 6.1 and 6.2 we compare performance of the fast summation algorithm against direct evaluation using (58). The tables are labeled as follows: $N$ is the number of particles, $T_{\text {apr }}$ is the time required to compute the initial approximations, 
TABle 6.1

Implementation in one dimension using B-splines of degree 3.

\begin{tabular}{|c|c|c|c|c||c|}
\hline$N$ & $T_{\text {apr }}$ & $T_{\text {cor }}$ & $T_{\text {alg }}$ & $T_{\text {dir }}$ & $E_{\infty}$ \\
\hline \hline 64 & $0.111 \mathrm{E}-03$ & $0.832 \mathrm{E}-04$ & $0.194 \mathrm{E}-03$ & $0.304 \mathrm{E}-03$ & $0.67627 \mathrm{E}-06$ \\
\hline 128 & $0.217 \mathrm{E}-03$ & $0.239 \mathrm{E}-03$ & $0.456 \mathrm{E}-03$ & $0.654 \mathrm{E}-03$ & $0.39181 \mathrm{E}-07$ \\
\hline 256 & $0.463 \mathrm{E}-03$ & $0.250 \mathrm{E}-03$ & $0.713 \mathrm{E}-03$ & $0.184 \mathrm{E}-02$ & $0.17899 \mathrm{E}-06$ \\
\hline 512 & $0.840 \mathrm{E}-03$ & $0.491 \mathrm{E}-03$ & $0.133 \mathrm{E}-02$ & $0.668 \mathrm{E}-02$ & $0.17084 \mathrm{E}-06$ \\
\hline 1024 & $0.181 \mathrm{E}-02$ & $0.938 \mathrm{E}-03$ & $0.275 \mathrm{E}-02$ & $0.381 \mathrm{E}-01$ & $0.11764 \mathrm{E}-06$ \\
\hline 2048 & $0.321 \mathrm{E}-02$ & $0.201 \mathrm{E}-02$ & $0.523 \mathrm{E}-02$ & $0.158 \mathrm{E}+00$ & $0.36255 \mathrm{E}-06$ \\
\hline 4096 & $0.743 \mathrm{E}-02$ & $0.412 \mathrm{E}-02$ & $0.116 \mathrm{E}-01$ & $0.640 \mathrm{E}+00$ & $0.29900 \mathrm{E}-06$ \\
\hline 8192 & $0.162 \mathrm{E}-01$ & $0.796 \mathrm{E}-02$ & $0.241 \mathrm{E}-01$ & $0.256 \mathrm{E}+01$ & $0.65214 \mathrm{E}-06$ \\
\hline
\end{tabular}

TABLE 6.2

Implementation in one dimension using B-splines of degree 11.

\begin{tabular}{|c|c|c|c|c||c|}
\hline$N$ & $T_{\text {apr }}$ & $T_{\text {cor }}$ & $T_{\text {alg }}$ & $T_{\text {dir }}$ & $E_{\infty}$ \\
\hline \hline 64 & $0.120 \mathrm{E}-03$ & $0.193 \mathrm{E}-03$ & $0.313 \mathrm{E}-03$ & $0.314 \mathrm{E}-03$ & $0.19390 \mathrm{E}-15$ \\
\hline 128 & $0.181 \mathrm{E}-03$ & $0.734 \mathrm{E}-03$ & $0.914 \mathrm{E}-03$ & $0.616 \mathrm{E}-03$ & $0.17910 \mathrm{E}-15$ \\
\hline 256 & $0.108 \mathrm{E}-02$ & $0.877 \mathrm{E}-03$ & $0.196 \mathrm{E}-02$ & $0.191 \mathrm{E}-02$ & $0.12462 \mathrm{E}-14$ \\
\hline 512 & $0.252 \mathrm{E}-02$ & $0.179 \mathrm{E}-02$ & $0.431 \mathrm{E}-02$ & $0.784 \mathrm{E}-02$ & $0.10585 \mathrm{E}-14$ \\
\hline 1024 & $0.534 \mathrm{E}-02$ & $0.379 \mathrm{E}-02$ & $0.913 \mathrm{E}-02$ & $0.400 \mathrm{E}-01$ & $0.45141 \mathrm{E}-15$ \\
\hline 2048 & $0.111 \mathrm{E}-01$ & $0.767 \mathrm{E}-02$ & $0.188 \mathrm{E}-01$ & $0.158 \mathrm{E}+00$ & $0.20654 \mathrm{E}-14$ \\
\hline 4096 & $0.279 \mathrm{E}-01$ & $0.153 \mathrm{E}-01$ & $0.432 \mathrm{E}-01$ & $0.639 \mathrm{E}+00$ & $0.30985 \mathrm{E}-14$ \\
\hline 8192 & $0.636 \mathrm{E}-01$ & $0.304 \mathrm{E}-01$ & $0.941 \mathrm{E}-01$ & $0.257 \mathrm{E}+01$ & $0.46730 \mathrm{E}-14$ \\
\hline
\end{tabular}

$T_{\text {cor }}$ is the time required to perform the correction step, $T_{\text {alg }}$ is the total time for the algorithm, and $T_{\text {dir }}$ is the time for direct computation using (58). The error is defined as

$$
E_{\infty}=\frac{\left\|\mathbf{g}-\mathbf{g}_{j}\right\|_{\infty}}{\|\mathbf{g}\|_{\infty}}
$$

where $\mathbf{g}$ represents the "exact" $N$-length vector computed with (58), $\mathbf{g}_{j}$ represents the approximation computed with the fast summation algorithm, and

$$
\|\mathbf{g}\|_{\infty}=\max _{1 \leq m \leq N}\left|g_{m}\right|
$$

The particle locations $\left\{x_{n}\right\}_{n=1}^{N}$ are generated randomly in $(0,1)$ and ordered such that $x_{n}<x_{n+1}$.

6.6. Summation algorithm in two dimensions. In this section we discuss implementation of the algorithm in two dimensions. We introduce an important feature, namely, singular value decomposition of the coefficient matrix, which reduces the two-dimensional algorithm to a sum of one-dimensional algorithms. The effectiveness of this strategy stems from the fact that the correction operator has a low separation rank.

The two-dimensional analogue of (48) is

$$
g_{m}=\sum_{\substack{n=1 \\ n \neq m}}^{N} K\left(x_{m}-x_{n}, y_{m}-y_{n}\right) f_{n}, \quad m=0,1, \ldots, N,
$$

where $\left(x_{n}, y_{n}\right) \in \mathbb{R}^{2}$ are particle locations. 
To approximate the kernel $K\left(x-x^{\prime}, y-y^{\prime}\right)$, we construct the multiresolution regularization of $K$,

$$
T_{j}\left(x, y, x^{\prime}, y^{\prime}\right)=\sum_{k, l} \phi_{j k}(x) \phi_{j l}(y) \sum_{k^{\prime}, l^{\prime}} t_{k-k^{\prime}, l-l^{\prime}}^{j} \phi_{j k^{\prime}}\left(x^{\prime}\right) \phi_{j l^{\prime}}\left(y^{\prime}\right)
$$

as described in section 3, using the two-dimensional two-scale difference equation (22) together with known asymptotic behavior of $K(x, y)$ as $\max \{|x|,|y|\} \rightarrow \infty$. Analogous to the one-dimensional estimate (54), there exists a constant $B_{j}$ such that, for each $\epsilon>0$, we have

$$
\left|K\left(x-x^{\prime}, y-y^{\prime}\right)-T_{j}\left(x, y, x^{\prime}, y^{\prime}\right)\right|<\epsilon
$$

if $\max \left\{\left|x-x^{\prime}\right|,\left|y-y^{\prime}\right|\right\}>B_{j}$ (see Appendix C).

6.6.1. Approximation step. The initial approximations have the form

$$
g_{j, m}=\sum_{n=1}^{N} T_{j}\left(x_{m}, y_{m}, x_{n}, y_{n}\right) f_{n}, \quad m=0,1, \ldots, N .
$$

Rearranging the sums, we obtain

$$
g_{j, m}=\sum_{k, l} \hat{s}_{k, l}^{j} \phi_{j, k}\left(x_{m}\right) \phi_{j, l}\left(y_{m}\right),
$$

where

$$
\hat{s}_{k, l}^{j}=\sum_{k^{\prime}, l^{\prime}} t_{k-k^{\prime}, l-l^{\prime}}^{j} s_{k^{\prime}, l^{\prime}}^{j}
$$

and

$$
s_{k^{\prime}, l^{\prime}}^{j}=\sum_{n=1}^{N} f_{n} \phi_{j, k^{\prime}}\left(x_{n}\right) \phi_{j, l^{\prime}}\left(y_{n}\right) .
$$

The operation indicated in (64) is accomplished using a two-dimensional FFT.

6.6.2. Correction step. As explained above it is necessary to correct errors in the initial approximation due to particles that are too close together at the chosen scale of resolution $j$. To accomplish this, we subtract the erroneous contribution from the nearby particles and add the correct contribution. Thus, it is necessary to evaluate the multiresolution kernel $T_{j}\left(x, y, x^{\prime}, y^{\prime}\right)$ in the region defined by $\max \left\{\left|x-x^{\prime}\right|,\left|y-y^{\prime}\right|\right\} \leq$ $B_{j}$. For this purpose we utilize the trigonometric expansion of $T_{j}$, as obtained in Appendix D. In two dimensions, the (already truncated) form is

$$
\begin{aligned}
2^{j(\alpha+2)} T_{j}\left(x_{m}, y_{m}, x_{n}, y_{n}\right) & \\
=I_{0,0} & +2 \sum_{k=1}^{3} I_{k, 0} \cos \left(2^{-j} k \pi\left(x_{m}+x_{n}\right)\right)+2 \sum_{l=1}^{3} I_{0, l} \cos \left(2^{-j} l \pi\left(y_{m}+y_{n}\right)\right) \\
& +4 \sum_{k=1}^{3} \sum_{l=1}^{3} I_{k, l} \cos \left(2^{-j} k \pi\left(x_{m}+x_{n}\right)\right) \cos \left(2^{-j} l \pi\left(y_{m}+y_{n}\right)\right),
\end{aligned}
$$


where

$$
I_{k, l}=I_{k, l}\left(2^{-j}\left(x_{m}-x_{n}\right), 2^{-j}\left(y_{m}-y_{n}\right)\right)
$$

for each pair of indices $(k, l)$, and

$$
I_{k, l}(x, y)=\sum_{i=-\infty}^{\infty} \sum_{j=-\infty}^{\infty}(-1)^{i k+j l} t_{i j} \Phi_{k}(x-i) \Phi_{l}(y-j)
$$

(see Appendix D for a definition of $\Phi_{k}$ ).

To facilitate computation of the functions $\left\{I_{k, l}\right\}$, it is advantageous to tabulate them, after first computing the singular value decomposition of the coefficient matrix. We obtain the expansions

$$
t_{i j}=\sum_{r=1}^{R} \sigma_{r} u_{i}^{(r)} v_{j}^{(r)}
$$

where $u_{i}^{(r)}$ and $v_{j}^{(r)}$ denote elements of the left and right singular vectors, respectively. The numerical rank $R$ is determined by truncating the expansion at the desired accuracy.

Substituting (68) into (67), we obtain

$$
I_{k, l}(x, y)=\sum_{r=1}^{R} \sigma_{r} \sum_{i}(-1)^{i k} u_{i}^{(r)} \Phi_{k}(x-i) \sum_{j}(-1)^{j l} v_{j}^{(r)} \Phi_{l}(y-j) .
$$

Let us define

$$
U_{k}^{(r)}(x)=\sum_{i} u_{i}^{(r)}(-1)^{i k} \Phi_{k}(x-i) \quad \text { and } \quad V_{l}^{(r)}(y)=\sum_{j} v_{j}^{(r)}(-1)^{j l} \Phi_{l}(y-j) ;
$$

then we can express $I_{k, l}$ as

$$
I_{k, l}(x, y)=\sum_{r=1}^{R} \sigma_{r} U_{k}^{(r)}(x) V_{l}^{(r)}(y) .
$$

Substituting (69) into (66), we obtain

$$
2^{j(\alpha+2)} T_{j}\left(x_{m}, y_{m}, x_{n}, y_{n}\right)=\sum_{r=1}^{R} \sigma_{r} U\left(x_{m}, x_{n}\right) V\left(y_{m}, y_{n}\right),
$$

where

$$
\begin{aligned}
& U(x, y)=U_{0}^{(r)}\left(2^{-j}(x-y)\right)+2 \sum_{k=1}^{3} \cos \left(2^{-j} k \pi(x+y)\right) U_{k}^{(r)}\left(2^{-j}(x-y)\right), \\
& V(x, y)=V_{0}^{(r)}\left(2^{-j}(x-y)\right)+2 \sum_{l=1}^{3} \cos \left(2^{-j} l \pi(x+y)\right) V_{l}^{(r)}\left(2^{-j}(x-y)\right) .
\end{aligned}
$$

Functions $\left\{U_{k}^{(r)}(x)\right\}$ and $\left\{V_{n}^{(r)}(y)\right\}$ are tabulated and stored in memory. Comparing (70) to (56), we see that the cost of evaluating the two-dimensional multiresolution kernel is $(2 \cdot R \cdot C)$, where $C$ is the cost of evaluating a one-dimensional kernel. 
TABLE 6.3

Implementation in two dimensions using B-splines of degree 3.

\begin{tabular}{|c|c|c|c|c||c|}
\hline$N$ & $T_{\text {apr }}$ & $T_{\text {cor }}$ & $T_{\text {alg }}$ & $T_{\text {dir }}$ & $E_{\infty}$ \\
\hline \hline 64 & $0.631 \mathrm{E}-03$ & $0.171 \mathrm{E}-03$ & $0.802 \mathrm{E}-03$ & $0.239 \mathrm{E}-03$ & $0.19049 \mathrm{E}-07$ \\
\hline 128 & $0.768 \mathrm{E}-03$ & $0.561 \mathrm{E}-03$ & $0.133 \mathrm{E}-02$ & $0.579 \mathrm{E}-03$ & $0.18717 \mathrm{E}-09$ \\
\hline 256 & $0.103 \mathrm{E}-02$ & $0.222 \mathrm{E}-02$ & $0.325 \mathrm{E}-02$ & $0.199 \mathrm{E}-02$ & $0.59922 \mathrm{E}-09$ \\
\hline 512 & $0.253 \mathrm{E}-02$ & $0.627 \mathrm{E}-02$ & $0.880 \mathrm{E}-02$ & $0.733 \mathrm{E}-02$ & $0.11588 \mathrm{E}-09$ \\
\hline 1024 & $0.810 \mathrm{E}-02$ & $0.140 \mathrm{E}-01$ & $0.221 \mathrm{E}-01$ & $0.395 \mathrm{E}-01$ & $0.50661 \mathrm{E}-09$ \\
\hline 2048 & $0.339 \mathrm{E}-01$ & $0.290 \mathrm{E}-01$ & $0.629 \mathrm{E}-01$ & $0.159 \mathrm{E}+00$ & $0.12780 \mathrm{E}-09$ \\
\hline 4096 & $0.397 \mathrm{E}-01$ & $0.118 \mathrm{E}+00$ & $0.158 \mathrm{E}+00$ & $0.642 \mathrm{E}+00$ & $0.58964 \mathrm{E}-10$ \\
\hline
\end{tabular}

6.7. An example in two dimensions. Let the kernel $K$ be defined by

$$
K(x, y)=\frac{1}{x^{2}+y^{2}}, \quad x^{2}+y^{2} \neq 0,
$$

which is homogeneous of degree zero. The discrete sums to be computed are

$$
g_{m}=\sum_{\substack{n=1 \\ n \neq m}}^{N} \frac{f_{n}}{\left(x_{m}-x_{n}\right)^{2}+\left(y_{m}-y_{n}\right)^{2}}, \quad 1 \leq m \leq N .
$$

The results are shown in Table 6.3, where $N$ is the number of particles, which were distributed randomly in the unit square $[0,1] \times[0,1]$. (Column headings and error definition are described in section 6.5.)

Appendix A. Classical regularization. Here we provide a complete derivation of the formulas (8a) and (8b) of section 2, as well as (33) in section 3.4. We begin with regularization of two functionals, which are defined in terms of the following expressions:

$$
x_{+}=\left\{\begin{array}{ll}
x, & x>0, \\
0, & x \leq 0
\end{array} \quad \text { and } \quad x_{-}=\left\{\begin{array}{cc}
|x|, & x<0 \\
0, & x \geq 0 .
\end{array}\right.\right.
$$

Using these expressions, we define the functionals

$$
\left(x_{+}^{\lambda}, \phi\right)=\int_{0}^{\infty} x^{\lambda} \phi(x) d x
$$

and

$$
\left(x_{-}^{\lambda}, \phi\right)=\int_{-\infty}^{0}|x|^{\lambda} \phi(x) d x=\int_{0}^{\infty} x^{\lambda} \phi(-x) d x,
$$

where $\phi(x)$ is an infinitely differentiable test function with compact support. Both integrals are convergent for $\operatorname{Re}(\lambda)>-1$.

The procedure used to obtain (6) of section 2 may be extended to define

$$
\begin{gathered}
\left(x_{+}^{\lambda}, \phi\right)=\int_{0}^{1} x^{\lambda}\left[\phi(x)-\phi(0)-x \phi^{\prime}(0)-\cdots-\frac{x^{n-1}}{(n-1) !} \phi^{(n-1)}(0)\right] d x \\
+\int_{1}^{\infty} x^{\lambda} \phi(x) d x+\sum_{k=0}^{n-1} \frac{\phi^{(k)}(0)}{k !(\lambda+k+1)},
\end{gathered}
$$


which may be viewed as an analytic continuation of the original functional (72a) to the region $\operatorname{Re}(\lambda)>-n-1, \lambda \neq-1,-2, \ldots,-n$, for any positive integer $n$. Similarly, we define

$$
\begin{aligned}
\left(x_{-}^{\lambda}, \phi\right)=\int_{0}^{1} x^{\lambda} & {\left[\phi(-x)-\sum_{k=0}^{n-1} \frac{\phi^{(k)}(0)}{k !}(-1)^{k} x^{k}\right] d x } \\
& +\int_{1}^{\infty} x^{\lambda} \phi(-x) d x+\sum_{k=0}^{n-1} \frac{\phi^{(k)}(0)}{k !(\lambda+k+1)}(-1)^{k},
\end{aligned}
$$

which is an analytic continuation of the functional (72b) into the region $\operatorname{Re}(\lambda)>$ $-n-1, \lambda \neq-1,-2, \ldots,-n$, for any positive integer $n$.

Our goal is to construct the generalized functions

$$
\left(x^{\lambda}, \phi\right)=\int_{-\infty}^{\infty} x^{\lambda} \phi(x) d x \quad \text { for } \lambda=-1,-2,-3, \ldots
$$

To this end, let us combine functionals (72a) and (72b) to obtain

$$
\left(x_{+}^{\lambda}-x_{-}^{\lambda}, \phi\right)=\int_{-\infty}^{\infty}|x|^{\lambda} \operatorname{sgn}(x) \phi(x) d x
$$

and

$$
\left(x_{+}^{\lambda}+x_{-}^{\lambda}, \phi\right)=\int_{-\infty}^{\infty}|x|^{\lambda} \phi(x) d x .
$$

To regularize (74a) and (74b), observe that in any strip $-n-1<\operatorname{Re}(\lambda)<-n$, equation (73a) may be expressed as

$$
\left(x_{+}^{\lambda}, \phi\right)=\int_{0}^{\infty} x^{\lambda}\left[\phi(x)-\sum_{k=0}^{n-1} \frac{\phi^{(k)}(0)}{k !} x^{k}\right] d x,
$$

since

$$
\int_{1}^{\infty} x^{\lambda+k} d x=-\frac{1}{\lambda+k+1} \quad \text { if } 0 \leq k \leq n-1 .
$$

Similarly, (73b) may be expressed as

$$
\left(x_{-}^{\lambda}, \phi\right)=\int_{0}^{\infty} x^{\lambda}\left[\phi(-x)-\sum_{k=0}^{n-1} \frac{\phi^{(k)}(0)}{k !}(-1)^{k} x^{k}\right] d x .
$$

Now let us replace $n$ by $2 m$, and add and subtract (75a) and (75b), to obtain

$$
\begin{aligned}
& \left(x_{+}^{\lambda}-x_{-}^{\lambda}, \phi\right)=\int_{0}^{\infty} x^{\lambda}\left[\phi(x)-\phi(-x)-2 \sum_{k=0}^{m-1} \frac{\phi^{(2 k+1)}(0)}{(2 k+1) !} x^{2 k+1}\right] d x \\
& \left(x_{+}^{\lambda}+x_{-}^{\lambda}, \phi\right)=\int_{0}^{\infty} x^{\lambda}\left[\phi(x)+\phi(-x)-2 \sum_{k=0}^{m-1} \frac{\phi^{(2 k)}(0)}{(2 k) !} x^{2 k}\right] d x
\end{aligned}
$$

The regularization of (74a) is defined by the expression (76a), which converges in the strip $-2 m-2<\operatorname{Re}(\lambda)<-2 m$, and the regularization of $(74 \mathrm{~b})$ is defined by the 
expression (76b), which converges in the strip $-2 m-1<\operatorname{Re}(\lambda)<-2 m+1$. In particular, we have

$$
\begin{aligned}
\left(x^{-2 m-1}, \phi\right) & =\int_{0}^{\infty} x^{-2 m-1}\left[\phi(x)-\phi(-x)-2 \sum_{k=1}^{m} \frac{\phi^{(2 k-1)}(0)}{(2 k-1) !} x^{2 k-1}\right] d x \\
\left(x^{-2 m}, \phi\right) & =\int_{0}^{\infty} x^{-2 m}\left[\phi(x)+\phi(-x)-2 \sum_{k=1}^{m} \frac{\phi^{(2 k-2)}(0)}{(2 k-2) !} x^{2 k-2}\right] d x
\end{aligned}
$$

For example, taking $m=0$ in the first expression and $m=1$ in the second, we obtain

$$
\begin{aligned}
\left(x^{-1}, \phi\right) & =\int_{0}^{\infty} \frac{\phi(x)-\phi(-x)}{x} d x \\
\left(x^{-2}, \phi\right) & =\int_{0}^{\infty} \frac{\phi(x)+\phi(-x)-2 \phi(0)}{x^{2}} d x .
\end{aligned}
$$

Multidimensional generalized functions, for example $r^{\lambda}$, where $r^{2}=x_{1}{ }^{2}+\cdots+x_{n}{ }^{2}$, can be regularized in similar fashion (see, e.g., [10] for details).

\section{Appendix B. Multiresolution analysis.}

B.1. Definition. The following definition is sufficient for our purposes (see, e.g., [9] or [15] for more general treatments).

DeFINITION 10. An MRA of $L^{2}\left(\mathbb{R}^{n}\right)$ is an increasing sequence $V_{j}, j \in \mathbb{Z}$, of closed linear subspaces of $L^{2}\left(\mathbb{R}^{n}\right)$ with the following properties:

$$
\bigcap_{-\infty}^{\infty} V_{j}=\{0\}, \quad \bigcup_{-\infty}^{\infty} V_{j} \text { is dense in } L^{2}\left(\mathbb{R}^{n}\right) ;
$$

for all $f \in L^{2}\left(\mathbb{R}^{n}\right)$ and all $j \in \mathbb{Z}$,

$$
f(x) \in V_{j} \Longleftrightarrow f(2 x) \in V_{j-1} ;
$$

for all $f \in L^{2}\left(\mathbb{R}^{n}\right)$ and all $k \in \mathbb{Z}^{n}$,

$$
f(x) \in V_{0} \Longleftrightarrow f(x-k) \in V_{0} ;
$$

there exists a function $\phi(x) \in V_{0}$ such that

$$
\left\{\phi(x-k) \mid k \in \mathbb{Z}^{n}\right\}
$$

forms an orthonormal basis for the space $V_{0}$.

The functions $\left\{\phi_{j, k}(x) \mid k \in \mathbb{Z}^{n}\right\}$ form an orthonormal basis for $V_{j}$, where

$$
\phi_{j, k}(x)=2^{-n j / 2} \phi\left(2^{-j} x-k\right) .
$$

Function $\phi(x)$ is called a scaling function, and a given MRA may have several different scaling functions. In this paper, for simplicity, we consider only compactly supported scaling functions that form an orthonormal basis for the MRA under consideration (however, see section 4). 
B.2. Tensor product construction. To construct an MRA for $L^{2}\left(\mathbb{R}^{n}\right)$, the simplest method is to form the tensor product of an MRA for $L^{2}(\mathbb{R})$ (see, e.g., [9], [15]). For example, if $\{\phi(x-k) \mid k \in \mathbb{Z}\}$ is a basis for $V_{0}$ in a one-dimensional MRA, then the tensor product $\left\{\phi\left(x_{1}-k_{1}\right) \phi\left(x_{2}-k_{2}\right) \mid\left(k_{1}, k_{2}\right) \in \mathbb{Z}^{2}\right\}$ is a basis for $V_{0}$ in a two-dimensional MRA, where $\left(x_{1}, x_{2}\right) \in \mathbb{R}^{2}$. This construction allows us to extend one-dimensional concepts to higher dimensions in a straightforward manner, and in what follows we confine our remarks to the one-dimensional case for simplicity.

B.3. Two-scale difference equation, vanishing moments. The scaling function satisfies a two-scale difference equation,

$$
\phi(x)=\sqrt{2} \sum h_{l} \phi(2 x-l),
$$

and $\phi$ is completely determined by the coefficients $\left\{h_{l}\right\}$ (see, e.g., [9]). Since $\phi$ is compactly supported, the number of nonzero coefficients in (82) is finite. Taking Fourier transforms on both sides of (82) we obtain

$$
\hat{\phi}(\xi)=m_{0}(\xi / 2) \hat{\phi}(\xi / 2),
$$

where

$$
m_{0}(\xi)=(1 / \sqrt{2}) \sum h_{l} e^{i l \xi}
$$

Each MRA has a given number of "vanishing moments," which determines the order of approximation available in the MRA. The number of vanishing moments equals the number of zeros of the function $m_{0}(\xi)$ at $\xi=\pi$. If there are $M$ vanishing moments, then $m_{0}$ has the form

$$
m_{0}(\xi)=\left[\left(1+e^{i \xi}\right) / 2\right]^{M} F(\xi)
$$

where $F$ is a $2 \pi$-periodic function such that $F(\pi) \neq 0$. In an MRA with $M$ vanishing moments, the identities

$$
\sum_{-\infty}^{\infty}(x-k)^{m} \phi(x-k)=\mu_{m}, \quad 0 \leq m \leq M-1,
$$

are satisfied by the scaling function $\phi$, where $\mu_{m}$ denotes the value of the integral

$$
\mu_{m}=\int_{-\infty}^{\infty} x^{m} \phi(x) d x
$$

B.4. Autocorrelation of the scaling function. The autocorrelation is defined by

$$
\Phi(x)=\int \phi(x+y) \phi(y) d y .
$$

It can be shown that $\Phi(x)$ satisfies the moments condition

$$
\int x^{m} \Phi(x) d x= \begin{cases}1, & m=0 \\ 0, & 1 \leq m \leq 2 M-1\end{cases}
$$


where $M$ is the number of vanishing moments in the MRA. (This result depends on orthonormality of functions $\{\phi(x-k)\}$.) Function $\Phi$ satisfies a two-scale difference equation,

$$
\Phi(x)=\sum_{m} a_{m} \Phi(2 x-m),
$$

where the coefficients $\left\{a_{m}\right\}$ may be obtained from the coefficients in (82),

$$
a_{k}=\sum_{l} h_{l} h_{l+k} .
$$

Function $\Phi$ is compactly supported, and the number of nonzero coefficients in (91) is finite. Thus there is a positive integer $m_{0}$ such that

$$
a_{m}=0 \quad \text { if }|m|>m_{0} .
$$

For an orthonormal system, we have

$$
a_{2 m}=\delta_{m, 0}, \quad \sum a_{2 m+1}=1,
$$

and from (91) it can be verified that

$$
a_{-m}=a_{m} .
$$

Appendix C. Estimate of $\left|\boldsymbol{K}-\boldsymbol{T}_{\boldsymbol{j}}\right|$. Let us estimate the difference between a homogeneous kernel $K(x-y)$ and its multiresolution regularization $T_{j}(x, y)$ on scale $j \in \mathbb{Z}$. The estimate holds for points $(x, y)$ such that the distance $|x-y|$ is sufficiently large, and follows from the observation that a homogeneous kernel is smooth away from the singularity at $x-y=0$. Smooth functions are well approximated in an MRA and, due to compact support of the basis functions, the singularity at the origin does not significantly affect the order of approximation in regions that do not contain the origin. The difference $\left|K(x-y)-T_{j}(x, y)\right| \rightarrow 0$ as $|x-y| \rightarrow \infty$ at a rate depending on the decay of the $M$ th derivative of $K(x)$ as $|x| \rightarrow \infty$, where $M$ is the number of vanishing moments in the MRA.

Proposition 11. Let $T_{j}(x, y)$ be the multiresolution regularization of a homogeneous kernel $K(x-y)$, where

$$
T_{j}(x, y)=\sum_{m} \sum_{n} t_{m-n}^{j} \phi_{j, m}(x) \phi_{j, n}(y) .
$$

Let $M$ be the number of vanishing moments in the MRA, and assume the scaling functions $\left\{\phi_{j, m}(x)\right\}$ are compactly supported and form an orthonormal basis. Then, for each $\epsilon>0$, there exists a constant $B_{j}$, which depends on the scale $j$, such that

$$
\left|T_{j}(x, y)-K(x-y)\right|<\epsilon \quad \text { if }|x-y|>B_{j} .
$$

Proof. Let $s$ be a positive, real number such that the support of $\phi(x)$ is contained in the interval $(-s, s)$; then

$$
\phi(x)=0 \quad \text { if }|x| \geq s .
$$


The coefficients $\left\{t_{m-n}^{j}\right\}$ in (95) are defined formally by

$$
t_{m-n}^{j}=\iint K(u-v) \phi_{j, m}(u) \phi_{j, n}(v) d u d v,
$$

and from (97) it follows that the integral in (98) converges if $|m-n|>2 s$. It follows also that, for a given $(x, y)$, the summation in (95) involves a finite number of terms. Let us impose the following condition:

$$
|x-y| \geq 2^{j}(4 s)+\delta, \quad \exists \delta>0 .
$$

Then all coefficients $t_{m-n}^{j}$ involved in the summation are defined by a convergent integral in (98).

For the remainder of the proof let the point $(x, y)$ be fixed and chosen such that (99) is satisfied. Substituting (98) into (95), we obtain

$$
\begin{aligned}
T_{j}(x, y) & =\sum_{m} \sum_{n} \phi_{j, m}(x) \phi_{j, n}(y) \iint K(u-v) \phi_{j, m}(u) \phi_{j, n}(v) d u d v \\
& =\iint K(u-v) P_{j}(x, u) P_{j}(y, v) d u d v,
\end{aligned}
$$

where we define

$$
P_{j}(x, y)=\sum_{k=-\infty}^{\infty} \phi_{j, k}(x) \phi_{j, k}(y)
$$

Expanding $K$ in a Taylor series about $(x-y)$, we have

$$
K(u-v)=\sum_{k=0}^{M-1} \frac{K^{(k)}(x-y)}{k !}[(u-v)-(x-y)]^{k}+R,
$$

where $R=R(u, v, x, y)$ is the remainder term. Let us perform the following computation with the truncated Taylor series $K-R$,

$$
\begin{aligned}
\iint & (K-R) P_{j}(x, u) P_{j}(y, v) d u d v \\
= & \sum_{k=0}^{M-1} \frac{K^{(k)}(x-y)}{k !} \sum_{l=0}^{k}\left(\begin{array}{l}
k \\
l
\end{array}\right)(-1)^{l} \delta_{k-l, 0} \delta_{l, 0}=K(x-y),
\end{aligned}
$$

where we have used the following identities (see, e.g., [15]);

$$
\int(x-y)^{k} P_{j}(x, y) d y=\delta_{k, 0} \quad \text { for } k=0,1, \ldots, M-1 .
$$

Combining (101) with (100) we have

$$
T_{j}(x, y)-K(x-y)=\iint R(x, y, u, v) P_{j}(x, u) P_{j}(y, v) d u d v
$$

and, since $R$ has the form

$$
R(x, y, u, v)=\frac{K^{(M)}(\xi-\eta)}{M !}[(u-v)-(x-y)]^{M},
$$


where $\xi$ is between $u$ and $x$, and $\eta$ is between $v$ and $y$, we obtain

$$
\left|T_{j}(x, y)-K(x-y)\right| \leq C_{1} \sup \frac{\left|K^{(M)}(\xi-\eta)\right|}{M !},
$$

where

$$
C_{1}=\iint\left|[(u-v)-(x-y)]^{M} P_{j}(x, u) P_{j}(y, v)\right| d u d v .
$$

Differentiating (10b) repeatedly, we obtain

$$
x^{M} K^{(M)}(x)=(\alpha+1)(\alpha+2) \cdots(\alpha+M) K(x),
$$

and, combining this with the explicit form of $K(x)$ given in (26), we obtain

$$
\frac{\left|K^{(M)}(\xi-\eta)\right|}{M !}=C_{2}|\xi-\eta|^{-M-1-\alpha},
$$

where $C_{2}$ is a constant. Because $\xi$ is constrained to lie between $x$ and $u$, and $\eta$ between $y$ and $v$, we find that

$$
|\xi-\eta| \geq|x-y|-2^{j}(4 s)=\delta>0 .
$$

Finally, given $\epsilon>0$, choose $\delta$ so large that

$$
C_{1} C_{2}|\xi-\eta|^{-M-1-\alpha}<\epsilon \text { if }|\xi-\eta|>\delta,
$$

and we have proved (96), with $B_{j}=2^{j}(4 s)+\delta(\epsilon)$.

Appendix D. Representation of kernel as trigonometric series. We derive an alternative expression for a multiresolution kernel $T_{j}(x, y)$ which provides a more efficient method for evaluation of the kernel at a given point. This alternative takes the form of a rapidly converging trigonometric series with variable coefficients. The coefficients are functions of the difference $(x-y)$ and, as functions of a single variable, are easily tabulated. Because the convergence is so rapid, we typically retain only the first four terms of the series to achieve the desired accuracy. This result does not depend on homogeneity of the original kernel $K$, nor on orthonormality of the scaling functions.

Recall the form of a multiresolution kernel on the scale $j=0$, namely,

$$
T_{0}(x, y)=\sum_{m} \sum_{n} t_{m-n} \phi(x-m) \phi(y-n),
$$

with some coefficients $\left\{t_{n}\right\}$.

Proposition 12. The kernel $T_{0}$ in (102) may be given expression as

$$
T_{0}(x, y)=\sum_{-\infty}^{\infty} e^{-i n \pi(x+y)} I_{n}(x-y)
$$

with

$$
I_{n}(z)=\left\{\begin{array}{cc}
\sum t_{k} \Phi_{n}(z-k) & \text { if } n \text { is even } \\
\sum(-1)^{k} t_{k} \Phi_{n}(z-k) & \text { if } n \text { is odd }
\end{array}\right.
$$


where

$$
\Phi_{n}(z)=\frac{1}{2 \pi} \int_{-\infty}^{\infty} e^{-i z \xi} \hat{\phi}(n \pi+\xi) \hat{\phi}(n \pi-\xi) d \xi
$$

and

$$
\hat{\phi}(\xi)=\int e^{i x \xi} \phi(x) d x, \quad \phi(x)=\frac{1}{2 \pi} \int e^{-i x \xi} \hat{\phi}(\xi) d \xi
$$

Remark 10. The expansion (103) converges at a rate governed by the rate of decay of $\hat{\phi}(\xi)$ and, in practice, we retain terms only up through $|n|=3$. Thus, (103) provides an efficient method for evaluating the kernel $T_{0}(x, y)$ at a point, if formulas for evaluating the $\Phi_{n}(z)$ are known. Explicit formulas (available from the authors) have been derived for the case in which the scaling function is a B-spline.

Proof. Let us begin by computing the two-dimensional Fourier transform,

$$
\begin{aligned}
\hat{T}_{0}(\xi, \eta) & =\sum_{m} \sum_{n} t_{m-n} \iint e^{i x \xi} e^{i y \eta} \phi(x-m) \phi(y-n) d x d y \\
& =\left(\sum_{m} \sum_{n} t_{m-n} e^{i m \xi} e^{i n \eta}\right) \hat{\phi}(\xi) \hat{\phi}(\eta) \\
& =\hat{t}(\xi)\left(\sum_{m} e^{i m(\xi+\eta)}\right) \hat{\phi}(\xi) \hat{\phi}(\eta),
\end{aligned}
$$

where we define the formal trigonometric series

$$
\hat{t}(\xi)=\sum_{-\infty}^{\infty} t_{k} e^{i k \xi} .
$$

Applying the inverse Fourier transform, we have

$$
T_{0}(x, y)=\frac{1}{(2 \pi)^{2}} \int e^{-i x \xi} \hat{t}(\xi) \hat{\phi}(\xi) \int e^{-i y \eta} \hat{\phi}(\eta)\left(\sum_{m} e^{i m(\xi+\eta)}\right) d \eta d \xi,
$$

and let us transform the inner integral as follows:

$$
\begin{aligned}
\int e^{-i y \eta} \hat{\phi}(\eta) & \left(\sum_{m} e^{i m(\xi+\eta)}\right) d \eta \\
& =\sum_{n=-\infty}^{\infty} \int_{2 \pi n}^{2 \pi(n+1)} e^{-i y \eta} \hat{\phi}(\eta)\left(\sum_{m} e^{i m(\xi+\eta)}\right) d \eta \\
& =\sum_{n=-\infty}^{\infty} \int_{0}^{2 \pi} e^{-i y(\eta+2 n \pi)} \hat{\phi}(\eta+2 n \pi)\left(\sum_{m} e^{i m(\xi+\eta)}\right) d \eta \\
& =2 \pi \sum_{n=-\infty}^{\infty} e^{-i y(-\xi+2 n \pi)} \hat{\phi}(-\xi+2 n \pi)
\end{aligned}
$$

where we have used the identity

$$
\sum_{m} e^{i m(\xi+\eta)}=2 \pi \delta(\xi+\eta)
$$


which holds in the sense of generalized functions on spaces of $2 \pi$-periodic functions. With this expression for the inner integral, we obtain

$$
\begin{aligned}
T_{0}(x, y) & =\frac{1}{2 \pi} \sum_{n=-\infty}^{\infty} e^{-2 \pi i n y} \int e^{-i \xi(x-y)} \hat{t}(\xi) \hat{\phi}(\xi) \hat{\phi}(-\xi+2 n \pi) d \xi \\
& =\frac{1}{2 \pi} \sum_{n=-\infty}^{\infty} e^{-2 \pi i n y} \int e^{-i(\xi+n \pi)(x-y)} \hat{t}(\xi+n \pi) \hat{\phi}(\xi+n \pi) \hat{\phi}(-\xi+n \pi) d \xi \\
& =\sum_{n=-\infty}^{\infty} e^{-i n \pi(x+y)} I_{n}(x-y)
\end{aligned}
$$

which establishes the result.

If the coefficients $\left\{t_{n}\right\}$ in (102) are obtained from regularization of a kernel homogeneous of degree $\alpha$, then the kernel in (103) also scales as in (16), namely,

$$
T_{j}(x, y)=2^{-\alpha j} \sum_{n=-\infty}^{\infty} e^{-i n \pi 2^{-j}(x-y)} 2^{-j} I_{n}\left(2^{-j}(x-y)\right) .
$$

If $\hat{\phi}(\xi)$ is an even function (for example, if $\phi$ is a central B-spline), then (103) can be rearranged to obtain

$$
T_{0}(x, y)=I_{0}(x-y)+2 \sum_{n=0}^{\infty} \cos (n \pi(x+y)) I_{n}(x-y) .
$$

In two dimensions, (103) has the form

$$
T_{0}\left(x, y, x^{\prime}, y^{\prime}\right)=\sum_{-\infty}^{\infty} \sum_{-\infty}^{\infty} I_{m, n}\left(x-x^{\prime}, y-y^{\prime}\right) e^{i n \pi\left(x+x^{\prime}\right)} e^{i m \pi\left(y+y^{\prime}\right)},
$$

where

$$
I_{m, n}(x, y)=\sum_{k=-\infty}^{\infty} \sum_{l=-\infty}^{\infty}(-1)^{m k+n l} t_{k, l} \Phi_{m}(x-k) \Phi_{n}(y-l)
$$

If $\hat{\phi}(\xi)$ is even, then

$$
\begin{aligned}
& T_{0}\left(x, y, x^{\prime}, y^{\prime}\right)= I_{0,0}\left(x-x^{\prime}, y-y^{\prime}\right) \\
&+2 \sum_{l=1}^{\infty} I_{0, l} \cos \left(l \pi\left(y+y^{\prime}\right)\right)+2 \sum_{k=1}^{\infty} I_{k, 0} \cos \left(k \pi\left(x+x^{\prime}\right)\right) \\
&+ 4 \sum_{k=1}^{\infty} \sum_{l=1}^{\infty} I_{k, l} \cos \left(k \pi\left(x+x^{\prime}\right)\right) \cos \left(l \pi\left(y+y^{\prime}\right)\right) . \\
& \text { REFERENCES }
\end{aligned}
$$

[1] B. K. Alpert, A class of bases in $L^{2}$ for the sparse representation of integral operators, SIAM J. Math. Anal., 24 (1993), pp. 246-262.

[2] B. Alpert, G. Beylkin, R. Coifman, And V. Rokhlin, Wavelet-like bases for the fast solution of second-kind integral equations, SIAM J. Sci. Comput., 14 (1993), pp. 159-184. 
[3] C. Anderson, A method of local corrections for computing the velocity field due to a distribution of vortex blobs, J. Comput. Phys., 62 (1986), pp. 111-123.

[4] G. BEYLKIN, On the representation of operators in bases of compactly supported wavelets, SIAM J. Numer. Anal., 6 (1992), pp. 1716-1740.

[5] G. Beylkin, R. Coifman, And V. Rokhlin, Fast wavelet transforms and numerical algorithms I, Comm. Pure Appl. Math., 44 (1991), pp. 141-183.

[6] H. Cheng, L. Greengard, and V. Rokhlin, A fast adaptive multipole algorithm in three dimensions, J. Comput. Phys., 155 (1999), pp. 468-498.

[7] C. K. ChuI, An Introduction to Wavelets, Academic Press, Boston, MA, 1992.

[8] A. Cohen, I. Daubechies, and J. Feauveau, Biorthogonal bases of compactly supported wavelets, Comm. Pure Appl. Math., 45 (1992), pp. 485-560.

[9] I. Daubechies, Ten Lectures on Wavelets, SIAM, Philadelphia, 1992.

[10] I. Gel'Fand and G. Shilov, Generalized Functions, Vol. 1, Academic Press, New York, 1964.

[11] L. Greengard and V. Rokhlin, A fast algorithm for particle simulations, J. Comput. Phys., 73 (1987), pp. 325-348.

[12] J. Jackson, Classical Electrodynamics, Wiley, New York, 1962.

[13] P. Kolm And V. Rokhlin, Numerical quadratures for singular and hypersingular integrals, Comput. Math. Appl., 41 (2001), pp. 327-352.

[14] W. LaWTON, Necessary and sufficient conditions for constructing orthonormal wavelet bases, J. Math. Phys., 1 (1991), pp. 57-61.

[15] Y. Meyer, Wavelets and Operators, Cambridge Stud. Adv. Math. 37, Cambridge University Press, Cambridge, 1992.

[16] L. Schwartz, Théorie des distributions, Hermann, Paris, 1966. 